\title{
Global Stability of Multigroup SIRS Epidemic Model with Varying Population Sizes and Stochastic Perturbation around Equilibrium
}

\author{
Xiaoming Fan \\ School of Mathematical Sciences, Harbin Normal University, Harbin 150500, China \\ Correspondence should be addressed to Xiaoming Fan; fanxm093@163.com
}

Received 8 October 2013; Accepted 17 December 2013; Published 20 January 2014

Academic Editor: Francisco Solís Lozano

Copyright (C) 2014 Xiaoming Fan. This is an open access article distributed under the Creative Commons Attribution License, which permits unrestricted use, distribution, and reproduction in any medium, provided the original work is properly cited.

\begin{abstract}
We discuss multigroup SIRS (susceptible, infectious, and recovered) epidemic models with random perturbations. We carry out a detailed analysis on the asymptotic behavior of the stochastic model; when reproduction number $\mathscr{R}_{0}>1$, we deduce the globally asymptotic stability of the endemic equilibrium by measuring the difference between the solution and the endemic equilibrium of the deterministic model in time average. Numerical methods are employed to illustrate the dynamic behavior of the model and simulate the system of equations developed. The effect of the rate of immunity loss on susceptible and recovered individuals is also analyzed in the deterministic model.
\end{abstract}

\section{Introduction}

To curb the spread and impact of viruses, it is important to study their feature, propagating methods, means, and limitation. Many studies about the outbreak and spread of disease have been done by means of establishing epidemic models. These researches provided some useful and valid reference for the characteristic of disease transmission. Based on these results of theoretical analysis, one can predict the future course of an outbreak and evaluate strategies to control an epidemic. In 1927, Kermack and McKendrick created an SIR model in which they considered a fixed population with only three compartments of three classes: susceptible $S(t)$, infected $I(t)$, and removed $R(t)$. $S(t)$ represents the number of individuals not yet infected with the disease at time $t$, or those susceptible to the disease. $I(t)$ denotes the number of individuals who have been infected with the disease and are capable of spreading the disease to those in the susceptible class. $R(t)$ is the compartment used for those individuals who have been infected and then removed from the disease, either due to immunization or due to death. Those in this class are not able to be infected again or to transmit the infection to others. A single group SIRS model is an extension of the SIR model. The SIRS model for infections that do not confer permanent immunity, that is, an infection that does not leave a long lasting immunity: thus individuals that have recovered return to being susceptible again, moving back into the $S(t)$ compartment. The only difference between SIR and SIRS is that SIRS model allows members of the recovered class to be free of infection and rejoin the susceptible class.

Considering different contact patterns, a distinct number of sexual partners, or different geography, and so forth, it is more appropriate to divide individual hosts into groups in modeling epidemic disease [1]. Therefore, it is reasonable to propose multigroup models to describe the transmission dynamics of viruses in heterogeneous host populations on epidemic models. In fact, there are already many scholars focusing their study on various forms of multigroup epidemic models (see [2-7]). They have also proved the global stability of the unique endemic equilibrium through Lyapunov function, which is one of the main mathematical challenges in analyzing multigroup models. Recently, Guo et al. have paid more attention to multigroup models [8-10]. They first proposed a graph-theoretic approach to the method 
of global Lyapunov functions and used it to establish the global stability of the interior equilibrium for more general models [8]. Muroya et al. considered a class of $n$-group $(n \geqslant 2)$ SIRS epidemic models described by the following system of equations [11]:

$$
\begin{gathered}
\dot{S}_{k}=\Lambda_{k}-d_{k}^{S} S_{k}-\sum_{j=1}^{n} \beta_{k j} S_{k}(t) I_{j}(t)+\delta_{k} R_{k}, \\
\dot{I}_{k}=\sum_{j=1}^{n} \beta_{k j} S_{k}(t) I_{j}(t)-\left(d_{k}^{I}+\gamma_{k}\right) I_{k}, \\
\dot{R}_{k}=\gamma_{k} I_{k}-\left(d_{k}^{R}+\delta_{k}\right) R_{k}, \quad k=1, \ldots, n .
\end{gathered}
$$

Nakata et al. [12] and Enatsu et al. [13] proposed an idea to extend Lyapunov functional techniques in McCluskey [14] for SIR epidemic models to SIRS epidemic models. By extending well-known Lyapunov function techniques, Muroya et al. [11] succeeded to prove the global stability of system (1) without use of the grouping technique by graph theory 4 in Guo et al. [10]. Note that because of environmental noises, the deterministic approach has some limitations in the mathematical modeling transmission of an infectious disease, several authors began to consider the effect of white noise in epidemic models [15-18]. Beretta et al. proved the stability of epidemic model with stochastic time delays influenced by probability under certain conditions [19]. Such type of stochastic perturbations firstly was proposed in $[19,20]$ and later was successfully used in many other papers for many other different systems (see, e.g., [21-27]). Yuan et al. in [28] and Yu et al. in [29] all investigated epidemic models with fluctuations around the positive equilibrium and they proved locally stochastically asymptotic stability of the positive equilibrium. Ji et al. also discuss a multigroup SIR model with stochastic perturbation and deduce the globally asymptotic stability of the disease-free equilibrium when $R_{0} \leq 1$, which means the disease will die out. When $R_{0}>1$, they derive the disease will prevail, which is measured through the difference between the solution and the endemic equilibrium of the deterministic model in time average [1]. Imhof and Walcher [30] considered a stochastic chemostat model and they proved that the stochastic model led to extinction even though the deterministic counterpart predicts persistence. In our previous work, we considered an SEIR epidemic model with constant immigration and random fluctuation around the endemic equilibrium, we carried out a detailed analysis on the asymptotic behavior of the stochastic model [31], and we also investigated a twogroup epidemic model with distributed delays and random perturbation [32]. In addition, we investigated multigroup SEIQR models with and without random perturbation in computer network [33]. In the present paper, based on system (1), to examine the influence of white noise on system (1) we also consider a stochastic version of the SIRS model by perturbing the deterministic system (1) by a white noise and assume that the perturbations are around the positive endemic equilibrium of epidemic models.

This paper is organized as follows. We begin in Section 2 with necessary background with respect to the deterministic multigroup SIRS model. We establish the global dynamics determined by the basic reproduction number $\mathscr{R}_{0}$ and introduce some results of graph theory used by Guo et al. We also review the important results in Theorem 2 on deterministic model (1) by Muroya et al. In Section 3 we derive the stochastic version from the deterministic model (1). In Section 4 we analyse the asymptotic behavior of the stochastic model by means of the method of Lyapunov functions and the theories of stochastic differential equation in Theorem 5. Numerical methods are employed to simulate the dynamic behavior of the model, and the effect of the rate of immunity loss on the recovered is also analyzed in the deterministic models and the corresponding stochastic models in Section 5. Finally, we give the conclusion of our paper in Section 6.

\section{Global Stability of Deterministic Multigroup SIRS Models}

First, let us review some theories and results on deterministic multigroup SIRS models; we summarize the parameters in the model (1) by the following list:

$\Lambda_{k}$ : the recruitment rate of the population,

$\beta_{k j}$ : transmission coefficient between compartments $S_{k}$ and $I_{j}$,

$d_{k}^{S}, d_{k}^{I}, d_{k}^{R}$ : the natural death rates of susceptible, infected, and recovered individuals in city $k$, respectively,

$\delta_{k}$ : the rate of immunity loss in the $k$ th group,

$\gamma_{k}$ : the natural recovery rate of the infected individuals in city $k$.

We assume $d_{k}^{S}, d_{k}^{I}, d_{k}^{R}, \Lambda_{k}>0$ and the rest of the parameters are nonnegative for all $k$. In particular, $\beta_{k j}=0$ if there is no transmission of the disease between compartments $S_{k}$ and $I_{j}$.

By the biological meanings, we may assume that

$$
d_{k}^{S} \leqslant \min \left\{d_{k}^{I}, d_{k}^{R}\right\}, \quad k=1,2, \ldots, n
$$

For each $k$, adding the three equations in (1) gives $\left(S_{k}+I_{k}+R_{k}\right)^{\prime} \leqslant \Lambda_{k}-d_{k}^{S}\left(S_{k}+I_{k}+R_{k}\right)$. Hence, $\limsup _{t \rightarrow \infty}\left(S_{k}+I_{k}+R_{k}\right) \leqslant \Lambda_{k} / d_{k}^{S}$. Therefore, omega limit sets of system (1) are contained in the following bounded region in the non-negative cone of $R^{3 n}$ :

$$
\begin{gathered}
\Gamma=\left\{\left(S_{1}, I_{1}, R_{1}, \ldots, S_{n}, I_{n}, R_{n}\right) \in R_{+}^{3 n} \mid S_{k} \leqslant S_{k}^{0},\right. \\
\left.S_{k}+I_{k}+R_{k} \leqslant \frac{\Lambda_{k}}{d_{k}^{S}}, 1 \leqslant k \leqslant n\right\} .
\end{gathered}
$$


It can be verified that region $\Gamma$ is positively invariant and system (1) always has the disease-free equilibrium $P_{0}=$ $\left(S_{1}^{0}, 0,0,0,0, \ldots, S_{n}^{0}, 0,0,0,0\right)$, where $S_{k}^{0}=\Lambda_{k} / d_{k}^{S}$ is the equilibrium of the $S_{k}$ population in the absence of disease $\left(I_{1}=I_{2}=\cdots=I_{n}=0\right)$. Let $\stackrel{\circ}{\Gamma}$ denote the interior of $\Gamma$. An endemic equilibrium $P^{*}=\left(S_{1}^{*}, I_{1}^{*}, R_{1}^{*}, \ldots, S_{n}^{*}, I_{n}^{*}, R_{n}^{*}\right)$ belongs to $\stackrel{\circ}{\Gamma}$ satisfying the equilibrium equations

$$
\begin{gathered}
\Lambda_{k}=\sum_{j=1}^{n} \beta_{k j} S_{k}^{*} I_{j}^{*}-d_{k}^{S} S_{k}^{*}+\delta_{k} R_{k}^{*}, \\
\left(d_{k}^{I}+\gamma_{k}\right) I_{k}^{*}=\sum_{j=1}^{n} \beta_{k j} S_{k}^{*} I_{j}^{*}, \\
\left(d_{k}^{I}+\delta_{k}\right) R_{k}^{*}=\gamma_{k} I_{k}^{*} .
\end{gathered}
$$

Let

$$
\mathscr{R}_{0}=\rho\left(M_{0}\right)
$$

denote the spectral radius of the matrix

$$
M_{0}=\left(\frac{\beta_{k j} S_{k}^{0}}{d_{k}^{I}+\gamma_{k}}\right)_{1 \leqslant k, j \leqslant n} .
$$

The parameter $\mathscr{R}_{0}$ is referred to as the basic reproduction number. Its biological significance is that if $\mathscr{R}_{0}<1$, the disease dies out while if $\mathscr{R}_{0}>1$, the disease becomes endemic [9]. In the following theorem, we show that the multi-group model (1) has at least one endemic equilibrium $P^{*}$ when $\mathscr{R}_{0}>$ 1 , and $P^{*}$ is globally stable [9].

The matrix $B=\left(\beta_{k j}\right)$ denotes the contact matrix. Associated with $B$, one can construct a directed graph $\mathfrak{L}=$ $G(B)$ whose vertex $k$ represents the $k$ th group, $k=1, \ldots, n$. A directed edge exists from vertex $k$ to vertex $j$ if and only if $\beta_{k j}>0$. Throughout the paper, we assume that $B$ is irreducible. This is equivalent to $G(B)$ being strongly connected. Biologically, this is the same as assuming that any two groups $k$ and $j$ have a direct or indirect route of transmission. More specifically, individuals in $I_{j}$ can infect ones in $S_{k}$ directly or indirectly. Now consider the linear system

$$
\overline{\mathfrak{B}} \ell=0 \text {, }
$$

where

$$
\overline{\mathfrak{B}}=\left[\begin{array}{cccc}
\sum_{i \neq 1} \bar{\beta}_{1 i} & -\bar{\beta}_{21} & \cdots & -\bar{\beta}_{n 1} \\
-\bar{\beta}_{12} & \sum_{i \neq 2} \bar{\beta}_{2 i} & \cdots & -\bar{\beta}_{n 2} \\
\vdots & \vdots & \ddots & \vdots \\
-\bar{\beta}_{1 n} & \bar{\beta}_{2 n} & \cdots & \sum_{i \neq n} \bar{\beta}_{n i}
\end{array}\right]
$$

and $\bar{\beta}_{k j}=\beta_{k j} S_{k}^{*} I_{j}^{*}, \bar{\beta}_{k j}>0,1 \leqslant k, j \leqslant n$. Let $\mathbb{Q}=G(B)$ denote the directed graph associated with matrix $B$ (and $\left(\bar{\beta}_{k j}\right)$ ), and let $C_{j k}$ denote the cofactor of the $(j, k)$ entry of $\overline{\mathfrak{B}}$.

We have the following fundamental lemma[8].
Lemma 1 (Kirchhoff's Matrix-Tree Theorem). Assume that $\left(\bar{\beta}_{k j}\right)_{n \times n}$ is irreducible and $n \geqslant 2$. Then the following results hold.

(1) The solution space of system (9) has dimension 1, with a basis $\left(\ell_{1}, \ell_{2}, \ldots, \ell_{n}\right)=\left(C_{11}, C_{22}, \ldots, C_{n n}\right)$.

(2) For $1 \leqslant k \leqslant n$,

$$
C_{k k}=\sum_{T \in \mathbb{T}_{k}} W(T)=\sum_{T \in \mathbb{T}_{k}} \prod_{(r, m) \in E(T)} \bar{\beta}_{r m}>0,
$$

where $\mathbb{\mathbb { T }}_{k}$ is the set of all directed spanning subtrees of $\mathbf{Q}$ that are rooted at vertex $k, W(T)$ is the weight of a directed tree $T$, and $E(T)$ denotes the set of directed arcs in a directed tree $T$.

Y. Muroya et al. proved the following theorem.

Theorem 2. Assume that $B=\left(\beta_{k j}\right)$ is irreducible and inequality (2) holds. If $\mathscr{R}_{0}>1$ and $d_{k}^{S} S^{*}-\delta_{k} R_{k}^{*} \geqslant 0$, then system (1) has at least one endemic equilibrium $P^{*}$ in $\stackrel{\circ}{\Gamma}$, and $P^{*}$ is globally asymptotically stable.

\section{Multigroup Stochastic SIRS Model}

Under assumptions that $\mathscr{R}_{0}>1, d_{k}^{S} S^{*}-\delta_{k} R_{k}^{*} \geqslant 0, d_{k}^{S} \leqslant$ $\min \left\{d_{k}^{I}, d_{k}^{R}\right\}$, and $\mathfrak{B}=\left(\beta_{k j}\right)$ is irreducible in Theorem 2, we know from Section 2 that there exists a positive endemic equilibrium $P^{*}$ in $\stackrel{\circ}{\Gamma}$. Furthermore, we assume stochastic perturbations are of white noise type, which are directly proportional to distances $S_{k}(t), I_{k}(t)$, and $R_{k}(t)$ from values of $S_{k}^{*}, I_{k}^{*}, R_{k}^{*}$, influence on the $S_{k}(t), I_{k}(t)$, and $R_{k}(t)$, respectively. So system (1) results in

$$
\begin{gathered}
\dot{S}_{k}=\Lambda_{k}-d_{k}^{S} S_{k}-\sum_{j=1}^{n} \beta_{k j} S_{k}(t) I_{j}(t)+\delta_{k} R_{k} \\
+\sigma_{1 k}\left(S_{k}-S_{k}^{*}\right) \dot{B}_{1 k}, \\
\dot{I}_{k}=\sum_{j=1}^{n} \beta_{k j} S_{k}(t) I_{j}(t)-\left(d_{k}^{I}+\gamma_{k}\right) I_{k}+\sigma_{2 k}\left(I_{k}-I_{k}^{*}\right) \dot{B}_{2 k}, \\
\dot{R}_{k}=\gamma_{k} I_{k}-\left(d_{k}^{R}+\delta_{k}\right) R_{k}+\sigma_{3 k}\left(R_{k}-R_{k}^{*}\right) \dot{B}_{3 k}, \\
k=1, \ldots, n,
\end{gathered}
$$

where $B_{1 k}(t), B_{2 k}(t)$, and $B_{3 k}(t)$ are independent standard Brownian motions and $\sigma_{i k}^{2}>0$ represent the intensities of $B_{i k}(t)(i=1,2,3)$, respectively. Obviously, stochastic system (12) has the same equilibrium points as system (1). Hence, when the stochastic model (12) satisfies the conditions of Theorem 2, we can ensure the existence of the positive equilibrium point of the stochastic model (12). Note that because of the influence of the white noise, the components of solutions to (12) can also be negative at some time. But because it is impossible for populations to be less than zero physically, once the negative sections appear on components of solutions of (12), we only need to consider the nonnegative 
section of the components of solutions. In the next section, we will investigate asymptotic stability of the equilibrium $P^{*}$ of system (12). To this end, we will construct a class of different Lyapunov functions to achieve our proof under certain conditions.

\section{Stochastic Stability of the Endemic Equilibrium}

Let us now proceed to discuss asymptotic stability of the system (12). In this paper, unless otherwise specified, let $\left(\Omega, \mathscr{F},\left\{\mathscr{F}_{t}\right\}_{t \geqslant t_{0}}, P\right)$ be a complete probability space with a filtration $\left\{\mathscr{F}_{t}\right\}_{t \geqslant t_{0}}$ satisfying the usual conditions (i.e., it is increasing and right continuous while $\mathscr{F}_{0}$ contains all $P$ null sets). Let $B_{i k}(t)$ be the Brownian motions defined on this probability space. If $\mathscr{R}_{0}>1$, then the stochastic system (12) can be centered at its endemic equilibrium $P^{*}=$ $\left(S_{1}^{*}, I_{1}^{*}, R_{1}^{*}, \ldots, S_{n}^{*}, I_{n}^{*}, R_{n}^{*}\right)$; by the change of variables

$$
\mathbf{u}_{k}=S_{k}-S_{k}^{*}, \quad \mathbf{v}_{k}=I_{k}-I_{k}^{*}, \quad \mathbf{w}_{k}=R_{k}-R_{k}^{*},
$$

we obtain the following system:

$$
\begin{aligned}
& \dot{\mathbf{u}}_{k}=-d_{k}^{S} \mathbf{u}_{k}-\sum_{j=1}^{n} \beta_{k j} S_{k}^{*} \mathbf{v}_{j}-\sum_{j=1}^{n} \beta_{k j} \mathbf{u}_{k} \mathbf{v}_{j} \\
&-\sum_{j=1}^{n} \beta_{k j} \mathbf{u}_{k} I_{j}^{*}+\delta_{k} \mathbf{w}_{k}+\sigma_{1 k} \mathbf{u}_{k} \dot{B}_{1 k}, \\
& \dot{\mathbf{v}}_{k}= \sum_{j=1}^{n} \beta_{k j} S_{k}^{*} \mathbf{v}_{j}+\sum_{j=1}^{n} \beta_{k j} \mathbf{u}_{k} \mathbf{v}_{j} \\
&+\sum_{j=1}^{n} \beta_{k j} \mathbf{u}_{k} I_{j}^{*}-\left(d_{k}^{I}+\gamma_{k}\right) \mathbf{v}_{k}+\sigma_{2 k} \mathbf{v}_{k} \dot{B}_{2 k}, \\
& \dot{\mathbf{w}}_{k}= \gamma_{k} \mathbf{v}_{k}-\left(d_{k}^{R}+\delta_{k}\right) \mathbf{w}_{k}+\sigma_{3 k} \mathbf{w}_{k} \dot{B}_{3 k}, \\
& k=1, \ldots, n .
\end{aligned}
$$

It is clear that the stability of equilibrium of system (12) is equivalent to the stability of zero solution of system (14). Considering the $d$-dimensional stochastic differential equation

$$
d \mathbf{x}(t)=f(\mathbf{x}(t), t) d t+g(\mathbf{x}(t), t) d B(t), \quad t \geqslant t_{0} .
$$

If the assumptions of the existence and uniqueness theorem are satisfied, then for any given initial value $\mathbf{x}\left(t_{0}\right)=x_{0} \in \mathbb{R}^{d}$, (15) has a unique global solution denoted by $\mathbf{x}\left(t ; t_{0}, x_{0}\right)$. For the purpose of stability, we assume in this section $f(\mathbf{0}, t)=0$ and $g(\mathbf{0}, t)=0$ for all $t \geqslant t_{0}$. So (15) admits a solution $\mathbf{x}(t) \equiv \mathbf{0}$ which is called the trivial solution or equilibrium position. We let $C^{2,1}\left(\mathbb{R}^{d} \times\left[t_{0}, \infty\right) ; \mathbb{R}_{+}\right)$denote the family of all nonnegative functions $V(\mathbf{x}, t)$ on $\mathbb{R}^{d} \times\left[t_{0}, \infty\right)$ which are continuously twice differentiable in $\mathbf{x}$ and once in $t$. Define the differential operator $L$ associated with (15) by

$$
L=\frac{\partial}{\partial t}+\sum_{i=1}^{d} f_{i}(\mathbf{x}, t) \frac{\partial}{\partial x_{i}}+\frac{1}{2} \sum_{i, j=1}^{d}\left[g^{T}(\mathbf{x}, t) g(\mathbf{x}, t)\right]_{i j} \frac{\partial^{2}}{\partial x_{i} x_{j}}
$$

If $L$ acts on a function $V \in C^{2,1}\left(\mathbb{R}^{d} \times\left[t_{0}, \infty\right) ; \mathbb{R}_{+}\right)$, then

$$
\begin{aligned}
L V(\mathbf{x}, t)= & V_{t}(\mathbf{x}, t)+V_{t}(\mathbf{x}, t) f(\mathbf{x}, t) \\
& +\frac{1}{2} \operatorname{trace}\left[g^{T}(\mathbf{x}, t) V_{x x}(\mathbf{x}, t) g(\mathbf{x}, t)\right] .
\end{aligned}
$$

Definition 3. (1) The trivial solution of (15) is said to be stochastically stable or stable in probability if for every pair of $\varepsilon \in(0,1)$ and $r>0$, there exists a $\delta=\delta\left(\varepsilon, r, t_{0}\right)>0$ such that

$$
P\left\{\left|\mathbf{x}\left(t ; t_{0}, x_{0}\right)\right|<r \forall t \geqslant t_{0}\right\} \geqslant 1-\varepsilon
$$

whenever $\left|x_{0}\right|<\delta$. Otherwise, it is said to be stochastically unstable.

(2) The trivial solution is said to be stochastically asymptotically stable if it is stochastically stable and for every $\varepsilon \in$ $(0,1)$, there exists a $\delta_{0}=\delta_{0}\left(\varepsilon, t_{0}\right)>0$ such that

$$
P\left\{\lim _{t \rightarrow \infty} \mathbf{x}\left(t ; t_{0}, x_{0}\right)=0\right\} \geqslant 1-\varepsilon
$$

whenever $\left|x_{0}\right|<\delta_{0}$.

(3) The trivial solution is said to be stochastically asymptotically stable in the large if it is stochastically stable and for all $x_{0} \in \mathbb{R}^{d}$

$$
P\left\{\lim _{t \rightarrow \infty} \mathbf{x}\left(t ; t_{0}, x_{0}\right)=0\right\}=1
$$

Before proving the main theorem we put forward a lemma in [34].

Lemma 4 (see [34]). If there exists a positive-definite decrescent radially unbounded function $V(\mathbf{x}, t) \in C^{2,1}\left(\mathbb{R}^{d} \times\right.$ $\left.\left[t_{0}, \infty\right) ; \mathbb{R}_{+}\right)$such that $L V(\mathbf{x}, t)$ is negative-definite, then the trivial solution of (15) is stochastically asymptotically stable in the large.

From the above lemma, we can obtain the stochastically asymptotical stability of equilibrium as following.

Theorem 5. Assume that $\mathfrak{B}=\left(\beta_{k j}\right)$ is irreducible and inequality (2) holds and $\mathscr{R}_{0}>1, d_{k}^{S} S_{k}^{*}-\delta_{k} R_{k}^{*} \geqslant 0$; then if the following condition is satisfied: 

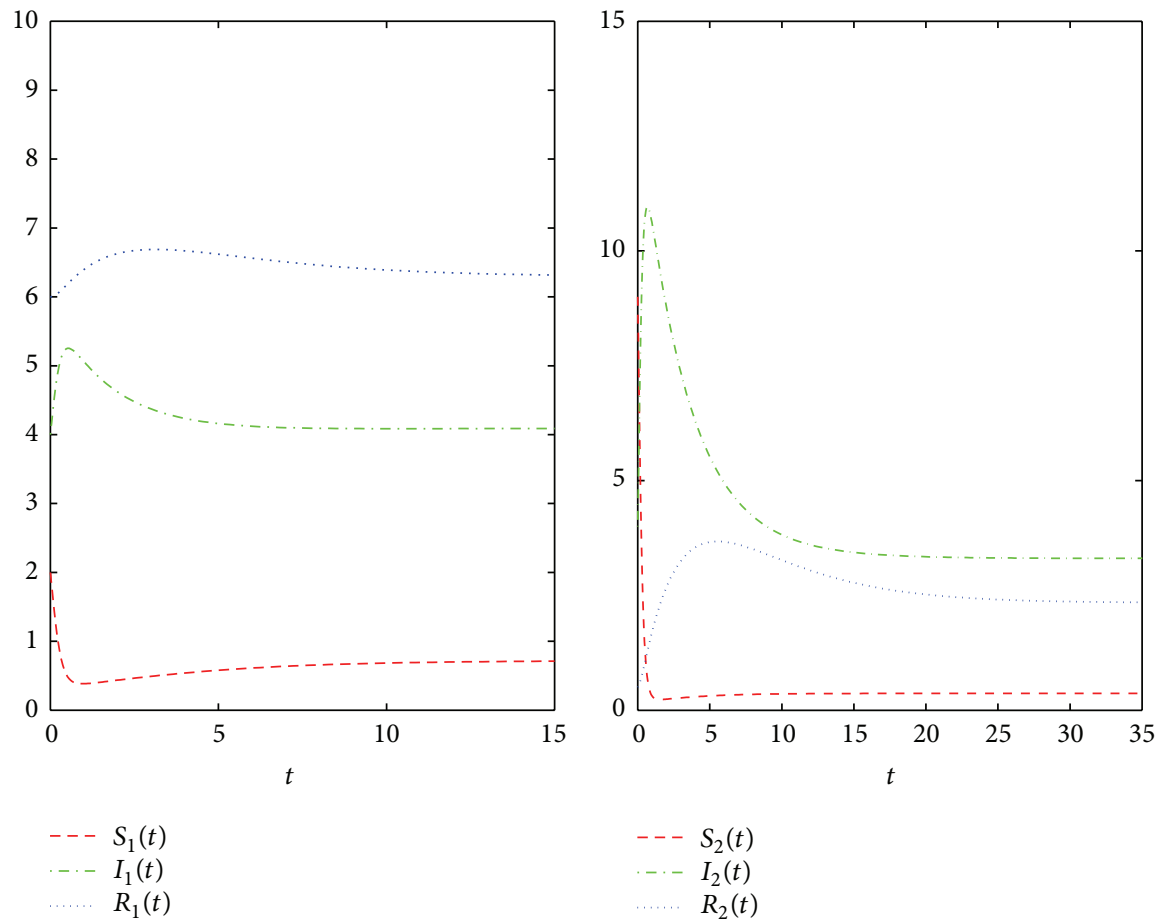

FIGURE 1: Deterministic trajectories of SIRS model (1) for initial condition $S_{1}(0)=2, I_{1}(0)=4, R_{1}(0)=6, S_{2}(0)=9, I_{2}(0)=4$, and $R_{2}(0)=0.5$.
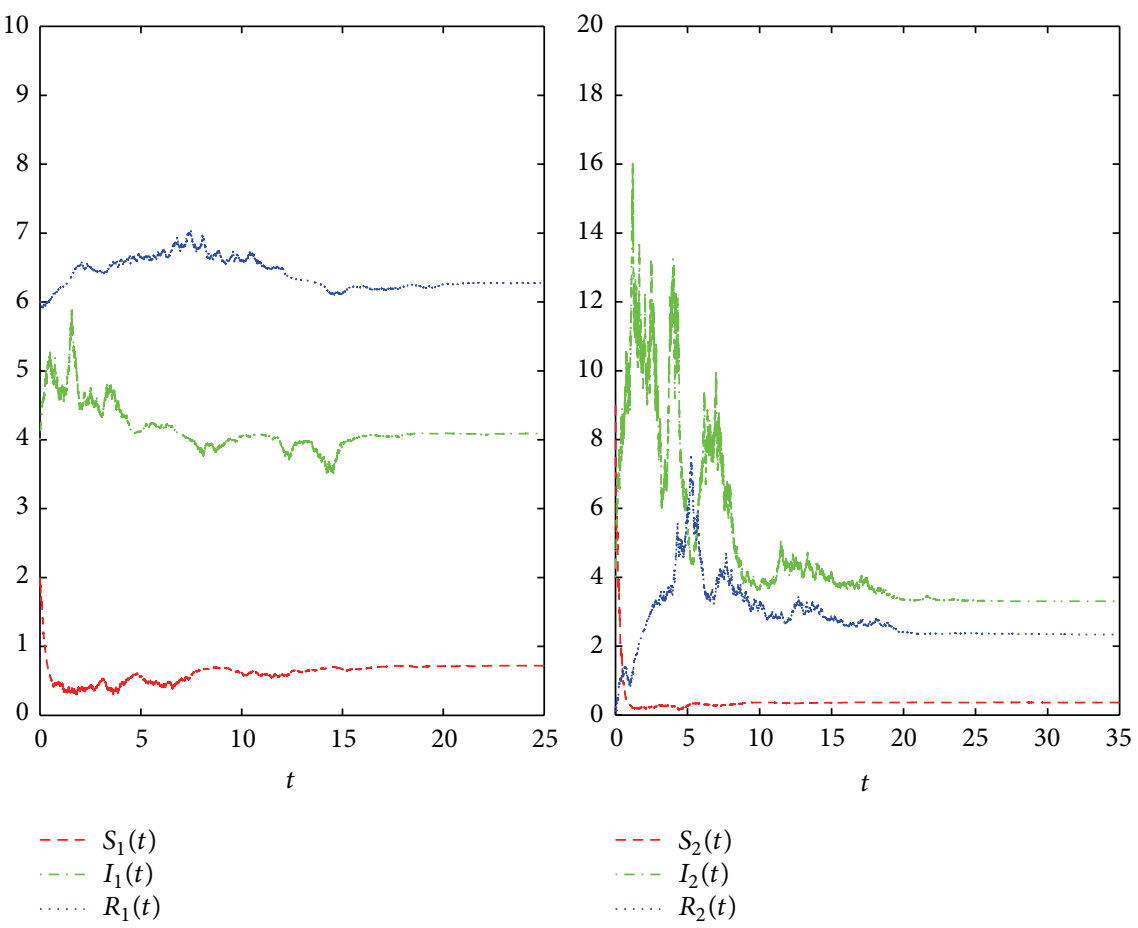

FIGURE 2: Stochastic trajectories of SIRS model (12) for $\sigma_{11}=0.4, \sigma_{21}=0.73, \sigma_{31}=0.45, \sigma_{12}=0.5, \sigma_{22}=0.67, \sigma_{32}=0.5$, and $\Delta t=10^{-3}$. 

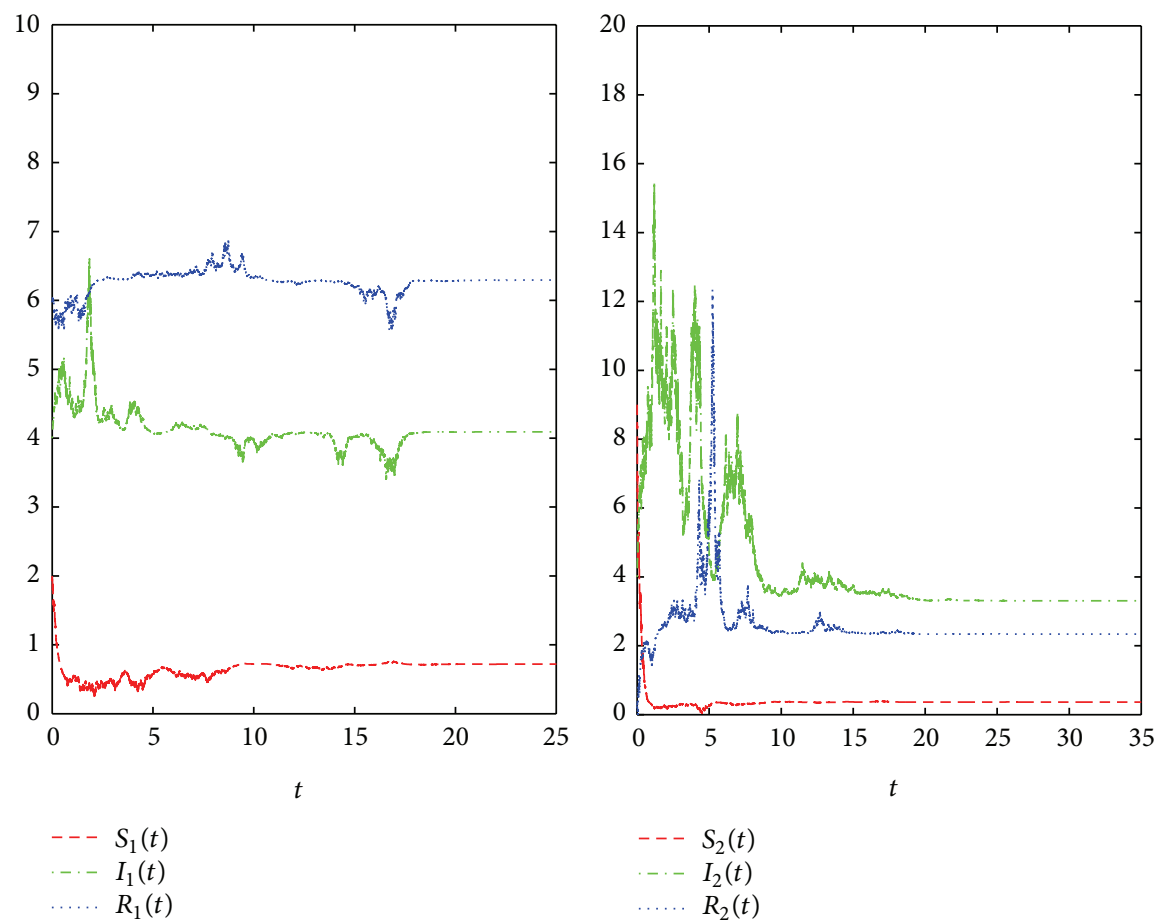

FIGURE 3: Stochastic trajectories of SIRS model (12) for $\sigma_{11}=0.7, \sigma_{21}=1.2, \sigma_{31}=1.3, \sigma_{12}=0.8, \sigma_{22}=0.8, \sigma_{32}=1.4$, and $\Delta t=10^{-3}$.
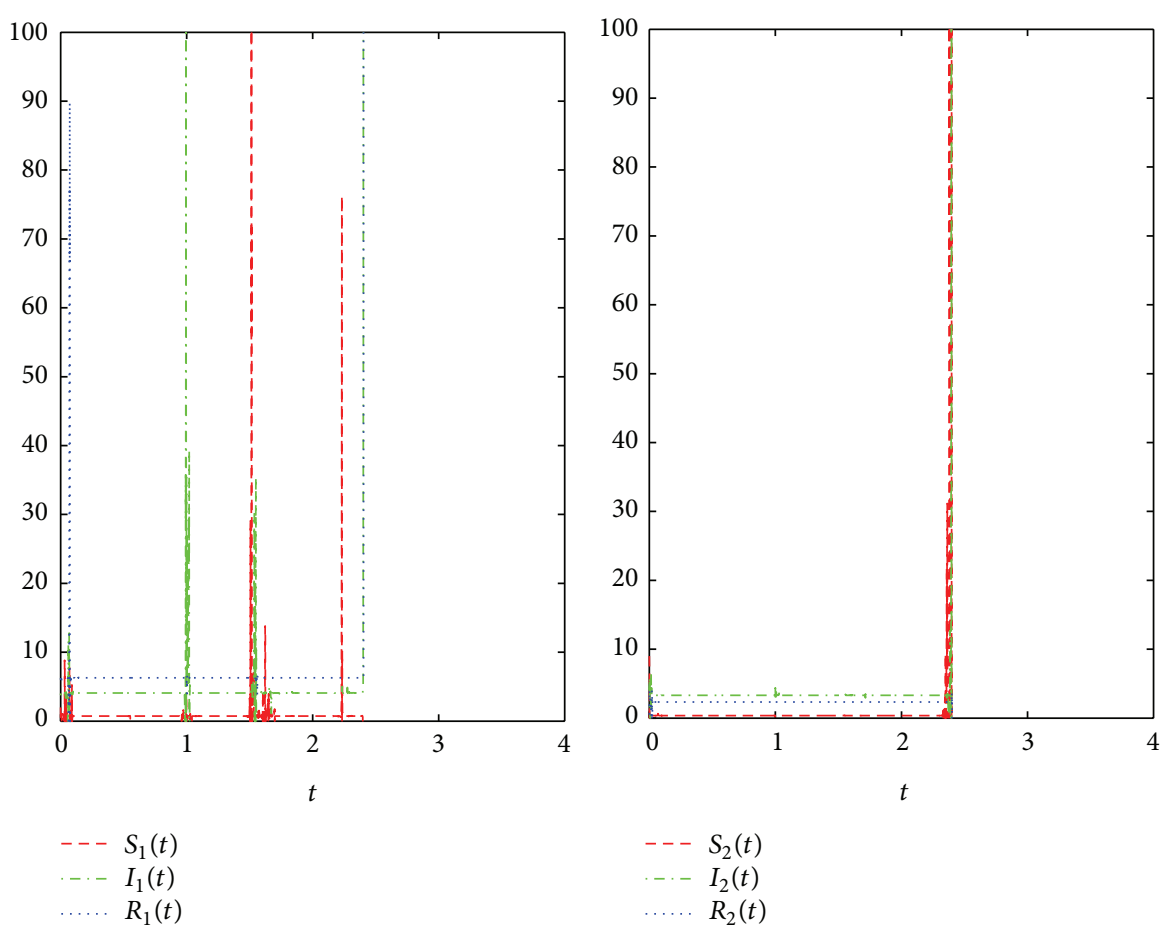

FIGURE 4: Stochastic trajectories of SIRS model (12) for $\sigma_{11}=40.5, \sigma_{21}=32.73, \sigma_{31}=36.92, \sigma_{12}=33.4, \sigma_{22}=39.67$, and $\sigma_{32}=30.5$. 

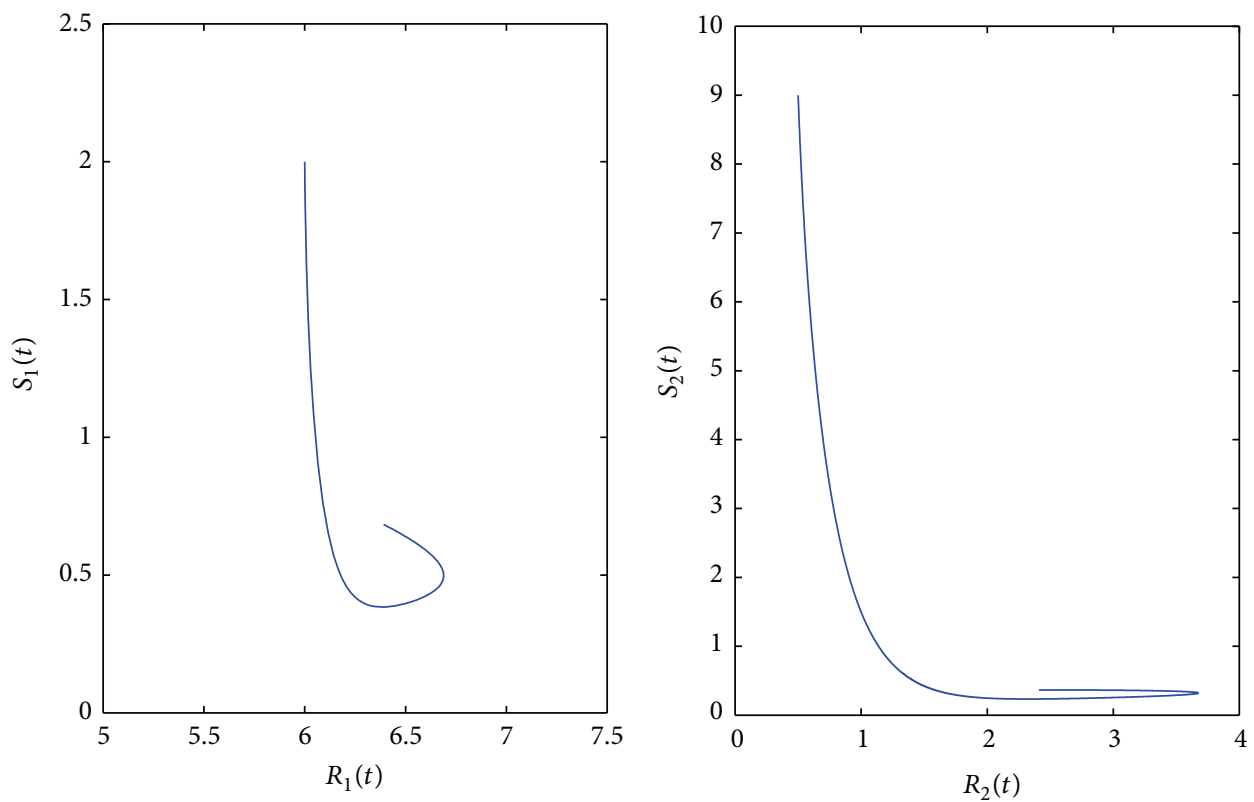

FigURE 5: The relation between $S_{k}$ and $R_{k}$ for the deterministic SIRS model.
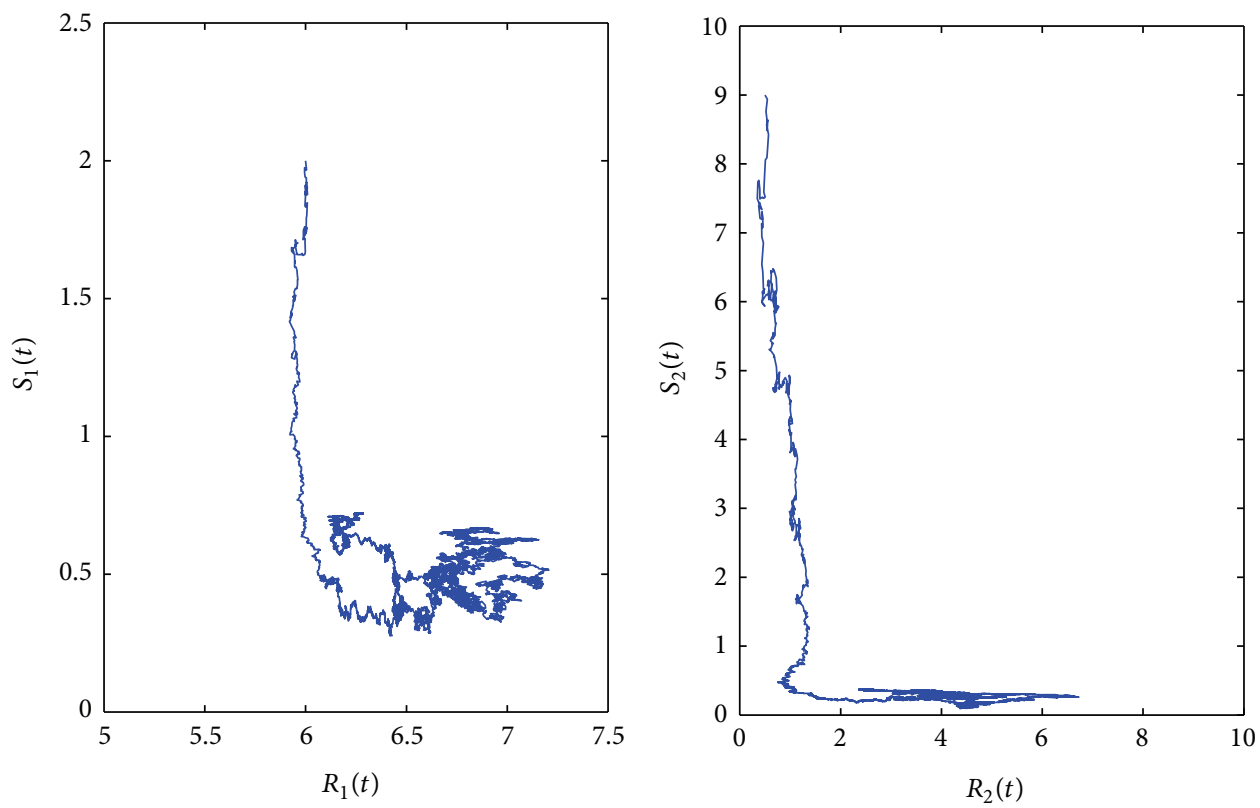

FIGURE 6: The relation between $S_{k}$ and $R_{k}$ for the stochastic SIRS model.

$$
\begin{array}{rlrl}
\sigma_{1 k}^{2}<2 d_{k}^{S}, \quad \sigma_{2 k}^{2}<\frac{2\left(d_{k}^{I}+\gamma_{k}\right) \sum_{j=1}^{n} \beta_{k j} I_{j}^{*}}{\sum_{j=1}^{n} \beta_{k j} I_{j}^{*}+d_{k}^{S}+d_{k}^{I}+\gamma_{k}}, & & +\frac{2 \delta_{k}^{2} \sum_{j=1}^{n} \beta_{k j} I_{j}^{*}}{M_{k}\left(d_{k}^{R}+\delta_{k}-(1 / 2) \sigma_{3 k}^{2}\right)} \\
\sigma_{3 k}^{2}<2\left(d_{k}^{R}+\delta_{k}\right), & <\frac{\left(d_{k}^{R}+\delta_{k}-(1 / 2) \sigma_{3 k}^{2}\right) M_{k}}{2 \gamma_{k}^{2} \sum_{j=1}^{n} \beta_{k j} I_{j}^{*}}, \\
\frac{\delta_{k}^{2}}{\left(d_{k}^{S}-(1 / 2) \sigma_{1 k}^{2}\right)\left(d_{k}^{R}+\delta_{k}-(1 / 2) \sigma_{3 k}^{2}\right)} &
\end{array}
$$



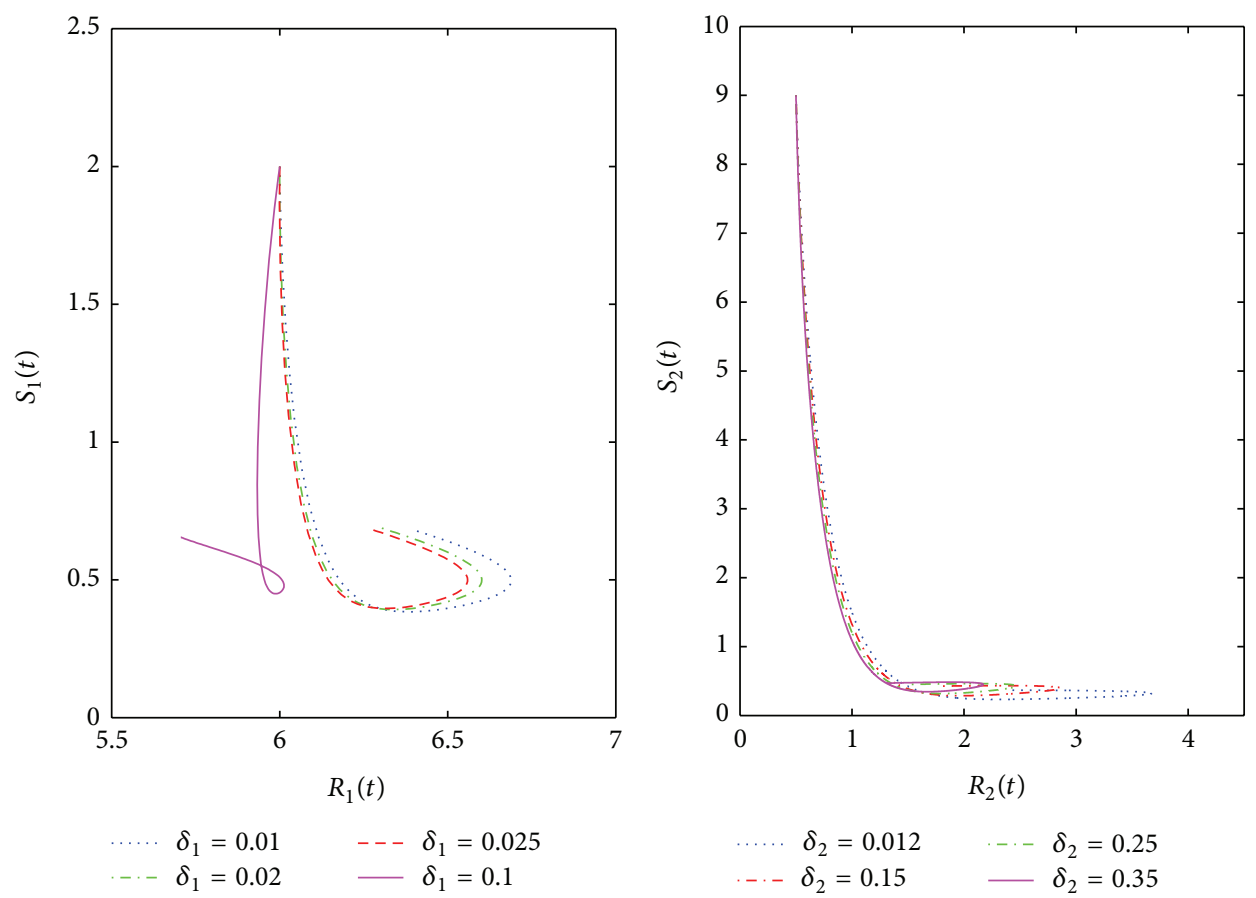

FIgURE 7: Comparison of relationships of $S_{k}$ and $R_{k}$ for the different values $\delta_{k}$ in the deterministic SIRS model.

the endemic equilibrium $P^{*}$ of system (12) is stochastically asymptotically stable in the large, where

$$
M_{k}=2\left(d_{k}^{I}+\gamma_{k}\right) \sum_{j=1}^{n} \beta_{k j} I_{j}^{*}-\left(\sum_{j=1}^{n} \beta_{k j} I_{j}^{*}+d_{k}^{S}+d_{k}^{I}+\gamma_{k}\right) \sigma_{2 k}^{2} .
$$

Proof. Set

$$
\overline{\mathfrak{B}}=\left[\begin{array}{cccc}
\sum_{i \neq 1} \bar{\beta}_{1 i} & -\bar{\beta}_{21} & \cdots & -\bar{\beta}_{n 1} \\
-\bar{\beta}_{12} & \sum_{i \neq 2} \bar{\beta}_{2 i} & \cdots & -\bar{\beta}_{n 2} \\
\vdots & \vdots & \ddots & \vdots \\
-\bar{\beta}_{1 n} & \bar{\beta}_{2 n} & \cdots & \sum_{i \neq n} \bar{\beta}_{n i}
\end{array}\right]
$$

and $\bar{\beta}_{k j}=\beta_{k j} S_{k}^{*} I_{j}^{*}, \bar{\beta}_{k j}>0,1 \leqslant k, j \leqslant n$.

Note that $\overline{\mathfrak{B}}$ is the Laplacian matrix of the matrix $\left(\bar{\beta}_{k j}\right)_{n \times n}$ (see Lemma 1). Since $\beta_{k j}$ is irreducible, the matrices $\left(\bar{\beta}_{k j}\right)_{n \times n}$ and $\overline{\mathfrak{B}}$ are also irreducible. Let $C_{k j}$ denote the cofactor of the $(k, j)$ entry of $\overline{\mathfrak{B}}$. We know that the system $\overline{\mathfrak{B} \ell}=0$ has a positive solution $\ell=\left(\ell_{1}, \ell_{2}, \ldots, \ell_{n}\right)$, where $\ell_{k}=C_{k k}>0$ for $k=1,2, \ldots, n$, by Lemma 1 .

It is easy to see that we only need to prove the zero solution of (14) is stochastically asymptotically stable in the large. Let $\mathbf{x}_{k}(t)=\left(\mathbf{u}_{k}(t), \mathbf{v}_{k}(t), \mathbf{w}_{k}(t)\right)^{T} \in R_{+}^{3}, k=1, \ldots, n$ and $\mathbf{x}(t)=$ $\left(\mathbf{x}_{1}(t), \ldots, \mathbf{x}_{n}(t)\right)^{T} \in R_{+}^{3 n}$. We define the Lyapunov function $V(\mathbf{x}(t))$ as follows:

$$
V(\mathbf{x})=\frac{1}{2} \sum_{k=1}^{n}\left(a_{k} \mathbf{v}_{k}^{2}+b_{k}\left(\mathbf{u}_{k}+\mathbf{v}_{k}\right)^{2}+c_{k} \mathbf{w}_{k}^{2}\right),
$$

where $a_{k}>0, b_{k}>0, c_{k}>0$ are real positive constants to be chosen later. Then it can be described as the quadratic form

$$
V(\mathbf{x})=\frac{1}{2} \sum_{k=1}^{n} \mathbf{x}_{k}^{T} Q \mathbf{x}_{k}
$$

where

$$
Q=\left(\begin{array}{ccc}
b_{k} & b_{k} & 0 \\
b_{k} & a_{k}+b_{k} & 0 \\
0 & 0 & c_{k}
\end{array}\right)
$$

is a symmetric positive-definite matrix. So it is obvious that $V(\mathbf{x})$ is positive-definite decrescent radially unbounded. For the sake of simplicity, (24) may be divided into three functions: $V(\mathbf{x})=V_{1}(\mathbf{x})+V_{2}(\mathbf{x})+V_{3}(\mathbf{x})$, where

$$
\begin{gathered}
V_{1}(\mathbf{x})=\frac{1}{2} a_{k} \mathbf{v}_{k}^{2}, \quad V_{2}(\mathbf{x})=\frac{1}{2} b_{k}\left(\mathbf{u}_{k}+\mathbf{v}_{k}\right)^{2}, \\
V_{3}(\mathbf{x})=\frac{1}{2} c_{k} \mathbf{w}_{k}^{2} .
\end{gathered}
$$


Using Itô’s formula and (5), we compute

$$
\begin{aligned}
L V_{1}=\sum_{k=1}^{n} a_{k} \mathbf{v}_{k}\left(\sum_{j=1}^{n} \beta_{k j} S_{k}^{*} \mathbf{v}_{j}+\sum_{j=1}^{n} \beta_{k j} \mathbf{u}_{k} \mathbf{v}_{j}\right. \\
\left.\quad+\sum_{j=1}^{n} \beta_{k j} \mathbf{u}_{k} I_{j}^{*}-\left(d_{k}^{I}+\gamma_{k}\right) \mathbf{v}_{k}\right)+\frac{1}{2} \sum_{k=1}^{n} a_{k} \sigma_{2 k}^{2} \mathbf{v}_{k}^{2}
\end{aligned}
$$$$
=\sum_{k=1}^{n} a_{k} \mathbf{v}_{k}\left(\sum_{j=1}^{n} \beta_{k j} S_{k}^{*} \mathbf{v}_{j}+\sum_{j=1}^{n} \beta_{k j} \mathbf{u}_{k} \mathbf{v}_{j}\right.
$$$$
\left.+\sum_{j=1}^{n} \beta_{k j} \mathbf{u}_{k} I_{j}^{*}-\frac{\sum_{j=1}^{n} \beta_{k j} S_{k}^{*} I_{j}^{*}}{I_{k}^{*}} \mathbf{v}_{k}\right)
$$$$
+\frac{1}{2} \sum_{k=1}^{n} a_{k} \sigma_{2 k}^{2} \mathbf{v}_{k}^{2}
$$$$
=\sum_{k=1}^{n} a_{k}\left(\sum_{j=1}^{n} \beta_{k j} S_{k}^{*} I_{k}^{*} I_{j}^{*} \frac{\mathbf{v}_{k}}{I_{k}^{*}} \frac{\mathbf{v}_{j}}{I_{j}^{*}}-\sum_{j=1}^{n} \beta_{k j} S_{k}^{*} I_{k}^{*} I_{j}^{*}\left(\frac{\mathbf{v}_{k}}{I_{k}^{*}}\right)^{2}\right)
$$$$
+\sum_{k=1}^{n} a_{k}\left(\sum_{j=1}^{n} \beta_{k j} \mathbf{u}_{k} \mathbf{v}_{k} \mathbf{v}_{j}+\sum_{j=1}^{n} \beta_{k j} \mathbf{u}_{k} \mathbf{v}_{k} I_{j}^{*}\right)
$$$$
+\frac{1}{2} \sum_{k=1}^{n} a_{k} \sigma_{2 k}^{2} \mathbf{v}_{k}^{2}
$$$$
=\sum_{k=1}^{n} a_{k}\left(\sum_{j=1}^{n} \bar{\beta}_{k j} I_{k}^{*} \frac{\mathbf{v}_{k}}{I_{k}^{*}} \frac{\mathbf{v}_{j}}{I_{j}^{*}}-\sum_{j=1}^{n} \bar{\beta}_{k j} I_{k}^{*}\left(\frac{\mathbf{v}_{k}}{I_{k}^{*}}\right)^{2}\right)
$$$$
+\sum_{k=1}^{n} a_{k}\left(\sum_{j=1}^{n} \beta_{k j} \mathbf{u}_{k} \mathbf{v}_{k} \mathbf{v}_{j}+\sum_{j=1}^{n} \beta_{k j} \mathbf{u}_{k} \mathbf{v}_{k} I_{j}^{*}\right)
$$$$
+\frac{1}{2} \sum_{k=1}^{n} a_{k} \sigma_{2 k}^{2} \mathbf{v}_{k}^{2}
$$$$
\leqslant \sum_{k=1}^{n} a_{k}\left(\frac{1}{2} \sum_{j=1}^{n} \bar{\beta}_{k j} I_{k}^{*}\left(\left(\frac{\mathbf{v}_{k}}{I_{k}^{*}}\right)^{2}+\left(\frac{\mathbf{v}_{j}}{I_{j}^{*}}\right)^{2}\right)\right.
$$$$
\left.-\sum_{j=1}^{n} \bar{\beta}_{k j} I_{k}^{*}\left(\frac{\mathbf{v}_{k}}{I_{k}^{*}}\right)^{2}\right)
$$$$
+\sum_{k=1}^{n} a_{k}\left(\sum_{j=1}^{n} \beta_{k j} \mathbf{u}_{k} \mathbf{v}_{k} \mathbf{v}_{j}+\sum_{j=1}^{n} \beta_{k j} \mathbf{u}_{k} \mathbf{v}_{k} I_{j}^{*}\right)+\frac{1}{2} \sum_{k=1}^{n} a_{k} \sigma_{2 k}^{2} \mathbf{v}_{k}^{2}
$$$$
=\frac{1}{2}\left(\sum_{k=1}^{n} a_{k} \sum_{j=1}^{n} \bar{\beta}_{k j} I_{k}^{*}\left(\frac{\mathbf{v}_{j}}{I_{j}^{*}}\right)^{2}-\sum_{k=1}^{n} \sum_{j=1}^{n} \bar{\beta}_{k j} I_{k}^{*}\left(\frac{\mathbf{v}_{k}}{I_{k}^{*}}\right)^{2}\right)
$$$$
+\sum_{k=1}^{n} a_{k}\left(\sum_{j=1}^{n} \beta_{k j} \mathbf{u}_{k} \mathbf{v}_{k} \mathbf{v}_{j}+\sum_{j=1}^{n} \beta_{k j} \mathbf{u}_{k} \mathbf{v}_{k} I_{j}^{*}\right)+\frac{1}{2} \sum_{k=1}^{n} a_{k} \sigma_{2 k}^{2} \mathbf{v}_{k}^{2} .
$$

Let $a_{k}=C_{k k} / I_{k}^{*}$, so $\ell_{k}=a_{k} I_{k}^{*}$. It follows from $\mathfrak{B} \ell=0$ and $\bar{\beta}_{j k}=\beta_{j k} S_{j}^{*} I_{k}^{*}$ that

$$
\sum_{j=1}^{n} \bar{\beta}_{j k} \ell_{j}=\sum_{k=1}^{n} \bar{\beta}_{k j} \ell_{k}
$$

which implies

$$
\sum_{k=1}^{n} a_{k} \sum_{j=1}^{n} \bar{\beta}_{k j} I_{k}^{*}\left(\frac{\mathbf{w}_{j}}{I_{j}^{*}}\right)^{2}=\sum_{k=1}^{n} a_{k} \sum_{j=1}^{n} \bar{\beta}_{k j} I_{k}^{*}\left(\frac{\mathbf{w}_{k}}{I_{k}^{*}}\right)^{2} .
$$

Hence, inequality (28) becomes

$$
\begin{aligned}
L V_{1} \leqslant & \sum_{k=1}^{n} a_{k}\left(\sum_{j=1}^{n} \beta_{k j} \mathbf{u}_{k} \mathbf{v}_{k} \mathbf{v}_{j}+\sum_{j=1}^{n} \beta_{k j} \mathbf{u}_{k} \mathbf{v}_{k} I_{j}^{*}\right) \\
& +\frac{1}{2} \sum_{k=1}^{n} a_{k} \sigma_{2 k}^{2} \mathbf{v}_{k}^{2} .
\end{aligned}
$$

Similarly, from Itô's formula, we obtain

$$
\begin{aligned}
L V_{2}= & \sum_{k=1}^{n} b_{k}\left(\mathbf{u}_{k}+\mathbf{v}_{k}\right)\left(-d_{k}^{S} \mathbf{u}_{k}-\left(d_{k}^{I}+\gamma_{k}\right) \mathbf{v}_{k}+\delta_{k} \mathbf{w}_{k}\right) \\
& +\frac{1}{2} \sum_{k=1}^{n} b_{k}\left(\sigma_{1 k}^{2} \mathbf{u}_{k}^{2}+\sigma_{2 k}^{2} \mathbf{v}_{k}^{2}\right) \\
= & -\sum_{k=1}^{n} b_{k}\left(d_{k}^{S}-\frac{1}{2} \sigma_{1 k}^{2}\right) \mathbf{u}_{k}^{2} \\
& -\sum_{k=1}^{n} b_{k}\left(d_{k}^{I}+\gamma_{k}-\frac{1}{2} \sigma_{2 k}^{2}\right) \mathbf{v}_{k}^{2} \\
& -\sum_{k=1}^{n} b_{k}\left(d_{k}^{S}+d_{k}^{I}+\gamma_{k}\right) \mathbf{u}_{k} \mathbf{v}_{k} \\
& +\sum_{k=1}^{n} b_{k} \delta_{k}\left(\mathbf{u}_{k}+\mathbf{v}_{k}\right) \mathbf{w}_{k}, \\
L V_{3}= & \sum_{k=1}^{n} c_{k} \mathbf{w}_{k}\left(\gamma_{k} \mathbf{v}_{k}-\left(d_{k}^{R}+\delta_{k}\right) \mathbf{w}_{k}\right) \\
& +\frac{1}{2} \sum_{k=1}^{n} c_{k} \sigma_{3 k}^{2} \mathbf{w}_{k}^{2} \\
= & -\sum_{k=1}^{n} c_{k}\left(d_{k}^{R}+\delta_{k}-\frac{1}{2} \sigma_{3 k}^{2}\right) \mathbf{w}_{k}^{2}+\sum_{k=1}^{n} c_{k} \gamma_{k} \mathbf{v}_{k} \mathbf{w}_{k} .
\end{aligned}
$$


Moreover, using Cauchy inequality, we can obtain

$$
\begin{aligned}
c_{k} \gamma_{k} \mathbf{v}_{k} \mathbf{w}_{k} \leqslant & \frac{1}{2} c_{k}\left(d_{k}^{R}+\delta_{k}-\frac{1}{2} \sigma_{3 k}^{2}\right) \mathbf{w}_{k}^{2} \\
& +\frac{c_{k} \gamma_{k}^{2}}{2\left(d_{k}^{R}+\delta_{k}-(1 / 2) \sigma_{3 k}^{2}\right)} \mathbf{v}_{k}^{2}, \\
b_{k} \delta_{k} \mathbf{u}_{k} \mathbf{w}_{k} \leqslant & \frac{1}{2} b_{k}\left(d_{k}^{S}-\frac{1}{2} \sigma_{1 k}^{2}\right) \mathbf{u}_{k}^{2} \\
& +\frac{b_{k} \delta_{k}^{2}}{2\left(d_{k}^{S}-(1 / 2) \sigma_{1 k}^{2}\right)} \mathbf{w}_{k}^{2},
\end{aligned}
$$

$b_{k} \delta_{k} \mathbf{v}_{k} \mathbf{w}_{k} \leqslant+\frac{b_{k}}{4 \sum_{j=1}^{n} \beta_{k j} I_{j}^{*}} M_{k} \mathbf{v}_{k}^{2}+\frac{b_{k} \delta_{k}^{2} \sum_{j=1}^{n} \beta_{k j} I_{j}^{*}}{M_{k}} \mathbf{w}_{k}^{2}$,

where

$$
\begin{aligned}
M_{k}= & 2\left(d_{k}^{I}+\gamma_{k}\right) \sum_{j=1}^{n} \beta_{k j} I_{j}^{*} \\
& -\left(\sum_{j=1}^{n} \beta_{k j} I_{j}^{*}+d_{k}^{S}+d_{k}^{I}+\gamma_{k}\right) \sigma_{2 k}^{2}
\end{aligned}
$$

Hence, we can calculate

$$
\begin{aligned}
& L V=L V_{1}+ L V_{2}+L V_{3} \\
&=\sum_{k=1}^{n}\left\{a_{k}\left(\sum_{j=1}^{n} \beta_{k j} \mathbf{u}_{k} \mathbf{v}_{k} \mathbf{v}_{j}+\sum_{j=1}^{n} \beta_{k j} \mathbf{u}_{k} \mathbf{v}_{k} I_{j}^{*}\right)\right. \\
&+\frac{1}{2} a_{k} \sigma_{2 k}^{2} \mathbf{v}_{k}^{2}-b_{k}\left(d_{k}^{S}-\frac{1}{2} \sigma_{1 k}^{2}\right) \mathbf{u}_{k}^{2} \\
&-b_{k}\left(d_{k}^{I}+\gamma_{k}-\frac{1}{2} \sigma_{2 k}^{2}\right) \mathbf{v}_{k}^{2}-b_{k}\left(d_{k}^{S}+d_{k}^{I}+\gamma_{k}\right) \mathbf{u}_{k} \mathbf{v}_{k} \\
&+b_{k} \delta_{k}\left(\mathbf{u}_{k}+\mathbf{v}_{k}\right) \mathbf{w}_{k}-c_{k}\left(d_{k}^{R}+\delta_{k}-\frac{1}{2} \sigma_{3 k}^{2}\right) \mathbf{w}_{k}^{2} \\
&\left.+c_{k} \gamma_{k} \mathbf{v}_{k} \mathbf{w}_{k}\right\} \\
& \leqslant \sum_{k=1}^{n}\left\{\begin{array}{l}
a_{k} \sum_{j=1}^{n} \beta_{k j} \mathbf{u}_{k} \mathbf{v}_{k} I_{j}^{*}+\frac{1}{2} a_{k} \sigma_{2 k}^{2} \mathbf{v}_{k}^{2} \\
-b_{k}\left(d_{k}^{S}-\frac{1}{2} \sigma_{1 k}^{2}\right) \mathbf{u}_{k}^{2}-b_{k}\left(d_{k}^{I}+\gamma_{k}-\frac{1}{2} \sigma_{2 k}^{2}\right) \mathbf{v}_{k}^{2}
\end{array}\right.
\end{aligned}
$$

$$
\begin{aligned}
& -b_{k}\left(d_{k}^{S}+d_{k}^{I}+\gamma_{k}\right) \mathbf{u}_{k} \mathbf{v}_{k}-c_{k}\left(d_{k}^{R}+\delta_{k}-\frac{1}{2} \sigma_{3 k}^{2}\right) \mathbf{w}_{k}^{2} \\
& +\frac{1}{2} c_{k}\left(d_{k}^{R}+\delta_{k}-\frac{1}{2} \sigma_{3 k}^{2}\right) \mathbf{w}_{k}^{2} \\
& +\frac{c_{k} \gamma_{k}^{2}}{2\left(d_{k}^{R}+\delta_{k}-(1 / 2) \sigma_{3 k}^{2}\right)} \mathbf{v}_{k}^{2} \\
& +\frac{1}{2} b_{k}\left(d_{k}^{S}-\frac{1}{2} \sigma_{1 k}^{2}\right) \mathbf{u}_{k}^{2}+\frac{b_{k} \delta_{k}^{2}}{2\left(d_{k}^{S}-(1 / 2) \sigma_{1 k}^{2}\right)} \mathbf{w}_{k}^{2} \\
& \left.+\frac{b_{k}}{4 \sum_{j=1}^{n} \beta_{k j} I_{j}^{*}} M_{k} \mathbf{v}_{k}^{2}+\frac{b_{k} \delta_{k}^{2} \sum_{j=1}^{n} \beta_{k j} I_{j}^{*}}{M_{k}} \mathbf{w}_{k}^{2}\right\}
\end{aligned}
$$$$
+\sum_{k=1}^{n} a_{k} \sum_{j=1}^{n} \beta_{k j} \mathbf{u}_{k} \mathbf{v}_{k} \mathbf{v}_{j}
$$$$
=\sum_{k=1}^{n}\left\{-\frac{1}{2} b_{k}\left(d_{k}^{S}-\frac{1}{2} \sigma_{1 k}^{2}\right) \mathbf{u}_{k}^{2}\right.
$$$$
+\left(a_{k} \sum_{j=1}^{n} \beta_{k j} I_{j}^{*}-b_{k}\left(d_{k}^{S}+d_{k}^{I}+\gamma_{k}\right)\right) \mathbf{u}_{k} \mathbf{v}_{k}
$$$$
+\frac{1}{2} a_{k} \sigma_{2 k}^{2} \mathbf{v}_{k}^{2}
$$$$
-b_{k}\left(d_{k}^{I}+\gamma_{k}-\frac{1}{2} \sigma_{2 k}^{2}\right) \mathbf{v}_{k}^{2}+\frac{b_{k}}{4 \sum_{j=1}^{n} \beta_{k j} I_{j}^{*}} M_{k} \mathbf{v}_{k}^{2}
$$$$
+\frac{c_{k} \gamma_{k}^{2}}{2\left(d_{k}^{R}+\delta_{k}-(1 / 2) \sigma_{3 k}^{2}\right)} \mathbf{v}_{k}^{2}
$$$$
-\frac{1}{2} c_{k}\left(d_{k}^{R}+\delta_{k}-\frac{1}{2} \sigma_{3 k}^{2}\right) \mathbf{w}_{k}^{2}
$$$$
\left.+\frac{b_{k} \delta_{k}^{2}}{2\left(d_{k}^{S}-(1 / 2) \sigma_{1 k}^{2}\right)} \mathbf{w}_{k}^{2}+\frac{b_{k} \delta_{k}^{2} \sum_{j=1}^{n} \beta_{k j} I_{j}^{*}}{M_{k}} \mathbf{w}_{k}^{2}\right\}
$$$$
+\sum_{k=1}^{n} a_{k} \sum_{j=1}^{n} \beta_{k j} \mathbf{u}_{k} \mathbf{v}_{k} \mathbf{v}_{j}
$$

Set

$$
a_{k}=\frac{\left(d_{k}^{S}+d_{k}^{I}+\gamma_{k}\right)}{\sum_{j=1}^{n} \beta_{k j} I_{j}^{*}} b_{k}
$$

then we have

$$
\begin{aligned}
L V \leqslant \sum_{k=1}^{n}\{ & -\frac{1}{2} b_{k}\left(d_{k}^{S}-\frac{1}{2} \sigma_{1 k}^{2}\right) \mathbf{u}_{k}^{2} \\
& -\frac{b_{k}}{2 \sum_{j=1}^{n} \beta_{k j} I_{j}^{*}}
\end{aligned}
$$




$$
\begin{aligned}
& \times\left(2\left(d_{k}^{I}+\gamma_{k}\right) \sum_{j=1}^{n} \beta_{k j} I_{j}^{*}\right. \\
& \left.-\left(\sum_{j=1}^{n} \beta_{k j} I_{j}^{*}+d_{k}^{S}+d_{k}^{I}+\gamma_{k}\right) \sigma_{2 k}^{2}\right) \mathbf{v}_{k}^{2} \\
& +\frac{b_{k}}{4 \sum_{j=1}^{n} \beta_{k j} I_{j}^{*}} M_{k} \mathbf{v}_{k}^{2}+\frac{c_{k} \gamma_{k}^{2}}{2\left(d_{k}^{R}+\delta_{k}-(1 / 2) \sigma_{3 k}^{2}\right)} \mathbf{v}_{k}^{2} \\
& -\frac{1}{2} c_{k}\left(d_{k}^{R}+\delta_{k}-\frac{1}{2} \sigma_{3 k}^{2}\right) \mathbf{w}_{k}^{2} \\
& +\frac{b_{k} \delta_{k}^{2}}{2\left(d_{k}^{S}-(1 / 2) \sigma_{1 k}^{2}\right)} \mathbf{w}_{k}^{2} \\
& \left.+\frac{b_{k} \delta_{k}^{2} \sum_{j=1}^{n} \beta_{k j} I_{j}^{*}}{M_{k}} \mathbf{w}_{k}^{2}\right\}+\sum_{k=1}^{n} a_{k} \sum_{j=1}^{n} \beta_{k j} \mathbf{u}_{k} \mathbf{v}_{k} \mathbf{v}_{j} \\
& =\sum_{k=1}^{n}\left\{-\frac{1}{2} b_{k}\left(d_{k}^{S}-\frac{1}{2} \sigma_{1 k}^{2}\right) \mathbf{u}_{k}^{2}-\frac{b_{k}}{4 \sum_{j=1}^{n} \beta_{k j} I_{j}^{*}} M_{k} \mathbf{v}_{k}^{2}\right. \\
& +\frac{c_{k} \gamma_{k}^{2}}{2\left(d_{k}^{R}+\delta_{k}-(1 / 2) \sigma_{3 k}^{2}\right)} \mathbf{v}_{k}^{2} \\
& -\frac{1}{2} c_{k}\left(d_{k}^{R}+\delta_{k}-\frac{1}{2} \sigma_{3 k}^{2}\right) \mathbf{w}_{k}^{2} \\
& \left.+\frac{b_{k} \delta_{k}^{2}}{2\left(d_{k}^{S}-(1 / 2) \sigma_{1 k}^{2}\right)} \mathbf{w}_{k}^{2}+\frac{b_{k} \delta_{k}^{2} \sum_{j=1}^{n} \beta_{k j} I_{j}^{*}}{M_{k}} \mathbf{w}_{k}^{2}\right\} \\
& +\sum_{k=1}^{n} a_{k} \sum_{j=1}^{n} \beta_{k j} \mathbf{u}_{k} \mathbf{v}_{k} \mathbf{v}_{j} \\
& =\sum_{k=1}^{n}\left\{-\frac{1}{2} b_{k}\left(d_{k}^{S}-\frac{1}{2} \sigma_{1 k}^{2}\right) \mathbf{u}_{k}^{2}\right. \\
& -\left(\frac{b_{k}}{4 \sum_{j=1}^{n} \beta_{k j} I_{j}^{*}} M_{k}\right. \\
& \left.-\frac{c_{k} \gamma_{k}^{2}}{2\left(d_{k}^{R}+\delta_{k}-(1 / 2) \sigma_{3 k}^{2}\right)}\right) \mathbf{v}_{k}^{2} \\
& -\left(\frac{1}{2} c_{k}\left(d_{k}^{R}+\delta_{k}-\frac{1}{2} \sigma_{3 k}^{2}\right)-\frac{b_{k} \delta_{k}^{2}}{2\left(d_{k}^{S}-(1 / 2) \sigma_{1 k}^{2}\right)}\right. \\
& \left.\left.-\frac{b_{k} \delta_{k}^{2} \sum_{j=1}^{n} \beta_{k j} I_{j}^{*}}{M_{k}}\right) \mathbf{w}_{k}^{2}\right\}+\sum_{k=1}^{n} a_{k} \sum_{j=1}^{n} \beta_{k j} \mathbf{u}_{k} \mathbf{v}_{k} \mathbf{v}_{j} \\
& =L V_{0}+\sum_{k=1}^{n} a_{k} \sum_{j=1}^{n} \beta_{k j} \mathbf{u}_{k} \mathbf{v}_{k} \mathbf{v}_{j}
\end{aligned}
$$

where

$$
\begin{aligned}
L V_{0}=\sum_{k=1}^{n}\{ & -\frac{1}{2} b_{k}\left(d_{k}^{S}-\frac{1}{2} \sigma_{1 k}^{2}\right) \mathbf{u}_{k}^{2} \\
& -\left(\frac{b_{k}}{4 \sum_{j=1}^{n} \beta_{k j} I_{j}^{*}} M_{k}\right. \\
& \left.-\frac{c_{k} \gamma_{k}^{2}}{2\left(d_{k}^{R}+\delta_{k}-(1 / 2) \sigma_{3 k}^{2}\right)}\right) \mathbf{v}_{k}^{2} \\
& -\left(\frac{1}{2} c_{k}\left(d_{k}^{R}+\delta_{k}-\frac{1}{2} \sigma_{3 k}^{2}\right)-\frac{b_{k} \delta_{k}^{2}}{2\left(d_{k}^{S}-(1 / 2) \sigma_{1 k}^{2}\right)}\right. \\
& \left.\left.-\frac{b_{k} \delta_{k}^{2} \sum_{j=1}^{n} \beta_{k j} I_{j}^{*}}{M_{k}}\right) \mathbf{w}_{k}^{2}\right\} .
\end{aligned}
$$

We let

$$
\begin{gathered}
\mathscr{A}_{k}=\frac{1}{2} b_{k}\left(d_{k}^{S}-\frac{1}{2} \sigma_{1 k}^{2}\right), \\
\mathscr{B}_{k}=\frac{b_{k}}{4 \sum_{j=1}^{n} \beta_{k j} I_{j}^{*}} M_{k}-\frac{c_{k} \gamma_{k}^{2}}{2\left(d_{k}^{R}+\delta_{k}-(1 / 2) \sigma_{3 k}^{2}\right)}, \\
\mathscr{C}_{k}=\frac{1}{2} c_{k}\left(d_{k}^{R}+\delta_{k}-\frac{1}{2} \sigma_{3 k}^{2}\right)-\frac{b_{k} \delta_{k}^{2}}{2\left(d_{k}^{S}-(1 / 2) \sigma_{1 k}^{2}\right)} \\
-\frac{b_{k} \delta_{k}^{2} \sum_{j=1}^{n} \beta_{k j} I_{j}^{*}}{M_{k}} .
\end{gathered}
$$

The proofs above show that if the condition (21) is satisfied, $\mathscr{A}_{k}, \mathscr{B}_{k}$, and $\mathscr{C}_{k}$ are positive constants. Let $\lambda=$ $\min _{k \in\{1, \ldots, n\}}\left\{\mathscr{A}_{k}, \mathscr{B}_{k}, \mathscr{C}_{k}\right\}$; then $\lambda>0$. From (37) and (38), one sees that

$$
\begin{aligned}
L V \leqslant & L V_{0}+\sum_{k=1}^{n} a_{k} \sum_{j=1}^{n} \beta_{k j} \mathbf{u}_{k} \mathbf{v}_{k} \mathbf{v}_{j} \\
= & -\sum_{k=1}^{n}\left(\mathscr{A}_{k} \mathbf{u}_{k}^{2}+\mathscr{B}_{k} \mathbf{v}_{k}^{2}+\mathscr{C}_{k} \mathbf{w}_{k}^{2}\right) \\
& +\sum_{k=1}^{n} a_{k} \sum_{j=1}^{n} \beta_{k j} \mathbf{u}_{k} \mathbf{v}_{k} \mathbf{v}_{j} \\
= & -\lambda \sum_{k=1}^{n}\left(\left|\mathbf{x}_{k}(t)\right|^{2}+o\left(\left|\mathbf{x}_{k}(t)\right|^{2}\right)\right) \\
= & -\lambda|\mathbf{x}(t)|^{2}+o\left(|\mathbf{x}(t)|^{2}\right),
\end{aligned}
$$

where $\left|\mathbf{x}_{k}(t)\right|=\sqrt{\mathbf{u}_{k}^{2}(t)+\mathbf{v}_{k}^{2}(t)+\mathbf{w}_{k}^{2}(t)}, \quad|\mathbf{x}(t)|=$ $\left(\sum_{k=1}^{n}\left|\mathbf{x}_{k}(t)\right|\right)^{1 / 2}$ and $o\left(|\mathbf{x}(t)|^{2}\right)$ is an infinitesimal of higher order of $|\mathbf{x}(t)|^{2}$ for $t \rightarrow \infty$. Hence, $L V(\mathbf{x}, t)$ is negativedefinite for $t \geqslant 0$. According to Lemma 4 , we therefore conclude that the zero solution of (12) is stochastically asymptotically stable in the large. The proof is complete. 


\section{Numerical Simulation}

Numerical methods are employed to solve the system (1) and (12) and to depict the behavior of the susceptible, infectious, and recovered nodes with respect to time. We numerically simulate the solution of system (1) and (12) with $n=2$. In this case, we have

$$
\begin{aligned}
& M_{0}=\left[\begin{array}{ll}
\frac{\beta_{11}\left(\Lambda_{1} / d_{1}^{S}\right)}{d_{1}^{I}+\gamma_{1}} & \frac{\beta_{12}\left(\Lambda_{1} / d_{1}^{S}\right)}{d_{1}^{I}+\gamma_{1}} \\
\frac{\beta_{21}\left(\Lambda_{2} / d_{2}^{S}\right)}{d_{2}^{I}+\gamma_{2}} & \frac{\beta_{22}\left(\Lambda_{2} / d_{2}^{S}\right)}{d_{2}^{I}+\gamma_{2}}
\end{array}\right]:=\left[\begin{array}{ll}
\beta_{11} K_{1} & \beta_{12} K_{1} \\
\beta_{21} K_{2} & \beta_{22} K_{2}
\end{array}\right], \\
& \mathscr{R}_{0} \\
& =\rho\left(M_{0}\right) \\
& =\frac{\beta_{11} K_{1}+\beta_{22} K_{2}+\sqrt{\left(\beta_{11} K_{1}-\beta_{22} K_{2}\right)^{2}+4 \beta_{12} \beta_{21} K_{1} K_{2}}}{2} .
\end{aligned}
$$

Let

$$
\begin{gathered}
\Lambda_{1}=2.5, \quad \beta_{11}=0.55, \quad \beta_{12}=0.35, \quad d_{1}^{S}=0.15, \\
d_{1}^{I}=0.2, \quad d_{1}^{R}=0.25, \quad \delta_{1}=0.01, \quad \gamma_{1}=0.4, \\
\Lambda_{2}=1.0, \quad \beta_{21}=0.5, \quad \beta_{22}=0.2, \quad d_{2}^{S}=0.1, \quad d_{2}^{I}=0.15, \\
d_{2}^{R}=0.2, \quad \delta_{2}=0.012, \quad \gamma_{2}=0.15 .
\end{gathered}
$$

Hence we obtain $S_{1}^{*}=0.7205, I_{1}^{*}=4.0914, R_{1}^{*}=6.2945$, $S_{2}^{*}=0.3663, I_{2}^{*}=3.3048, R_{2}^{*}=2.3383$. We can calculate

$$
\begin{gathered}
\mathscr{R}_{0}=\frac{250}{9}>1, \quad d_{1}^{S} S_{1}^{*}-\delta_{1} R_{1}^{*}=0.04513>0, \\
d_{2}^{S} S_{2}^{*}-\delta_{2} R_{2}^{*}=0.0085704>0 .
\end{gathered}
$$

In the absence of noise, we simulate the global stability of the endemic equilibrium of deterministic system (1) in Figure 1, and we always choose initial value $S_{1}(0)=2.0, I_{1}(0)=4.0$, $R_{1}(0)=6.0, S_{2}(0)=9.0, I_{2}(0)=4.0$, and $R_{2}(0)=0.5$.
Next, we consider the effect of stochastic fluctuations of environment to the endemic equilibrium of the corresponding deterministic system. Given the discretization of system (12) for $t=0, \Delta t, 2 \Delta t, \ldots, n \Delta t$, and $k=1,2$,

$$
\begin{aligned}
S_{k, i+1}= & S_{k, i}+\left(\Lambda_{k}-d_{k}^{S} S_{k, i}-\beta_{k 1} S_{k, i} I_{1, i}\right. \\
& \left.-\beta_{k 2} S_{k, i} I_{2, i}+\delta_{k} R_{k, i}\right) \Delta t \\
& +\sigma_{1 k}\left(S_{k, i}-S_{k}^{*}\right) \sqrt{\Delta t} \varepsilon_{1 k, i}, \\
I_{k, i+1}= & I_{k, i}+\left(\beta_{k 1} S_{k, i} I_{1, i}+\beta_{k 2} S_{k, i} I_{2, i}-\left(d_{k}^{I}+\gamma_{k}\right) I_{k, i}\right) \Delta t \\
& +\sigma_{2 k}\left(I_{k, i}-I_{k}^{*}\right) \sqrt{\Delta t} \varepsilon_{2 k, i}, \\
R_{k, i+1}= & R_{k, i}+\left(\gamma_{k} I_{k, i}-\left(d_{k}^{I}+\delta_{k}\right) R_{k, i}\right) \Delta t \\
& +\sigma_{3 k}\left(R_{k, i}-R_{k}^{*}\right) \sqrt{\Delta t} \varepsilon_{3 k, i},
\end{aligned}
$$

where time increment $\Delta t>0$, and $\varepsilon_{1 k, i}, \varepsilon_{2 k, i}, \varepsilon_{3 k, i}$ are $N(0,1)$-distributed independent random variables which can be generated numerically by pseudorandom number generators.

As mentioned above, there is an endemic equilibrium $P^{*}=\left(S_{1}^{*}, I_{1}^{*}, R_{1}^{*}, S_{2}^{*}, I_{2}^{*}, R_{2}^{*}\right)$ of system (1) when $\mathscr{R}_{0}>1$, where

$$
\begin{array}{lll}
S_{1}^{*}=0.7205, & I_{1}^{*}=4.0914, & R_{1}^{*}=6.2945, \\
S_{2}^{*}=0.3663, & I_{2}^{*}=3.3048, & R_{2}^{*}=2.3383 .
\end{array}
$$

Figure 2 corresponds to $\sigma_{11}=0.4, \sigma_{21}=0.73, \sigma_{31}=0.45$, $\sigma_{12}=0.5, \sigma_{22}=0.67$, and $\sigma_{32}=0.5$; the numerical simulation shows that the endemic equilibrium of stochastic system (12) is global asymptotically stable under the condition (21), while Figure 3 corresponds to $\sigma_{11}=0.7, \sigma_{21}=1.2, \sigma_{31}=1.3$, $\sigma_{12}=0.8, \sigma_{22}=0.8$, and $\sigma_{32}=1.4$. Moreover, comparison of Figures 2 and 3 suggests that fluctuations enhance as the noise level increases. Note that the condition (21) is just a sufficient condition. When this condition is not satisfied, the stochastic system (12) may be or may not be stable. For instance, the intensities of Brownian motions $\sigma_{11}=0.7$, $\sigma_{21}=1.2, \sigma_{31}=1.3, \sigma_{12}=0.8, \sigma_{22}=0.8$, and $\sigma_{32}=$ 1.4 do not meet the condition (21), but we can see from Figure 3 that the stochastic system (12) is still asymptotically stable. If we choose $\sigma_{11}=40.5, \sigma_{21}=32.73, \sigma_{31}=36.92$, $\sigma_{12}=33.4, \sigma_{22}=39.67$, and $\sigma_{32}=30.5$, then the solution of the stochastic system (12) is not asymptotically stable but explodes to infinity at the finite time (see Figure 4).

The relationships between $S_{k}$ and $R_{k}$ are also observed and are depicted in Figures 5 and 6 . Note that these two curves of Figure 5 show that the number of susceptible individuals sharply declines to near $S_{k}^{*}$ as the number of the recovery individuals increases slightly and then increases with the number of the increasing recovered individuals gently and goes on increasing with the number of the decreasing recovered individuals, and finally both reach the steady state values. For the stochastic version, Figure 6 corresponds to $\sigma_{11}=0.4$, $\sigma_{21}=0.73, \sigma_{31}=0.45, \sigma_{12}=0.5, \sigma_{22}=0.67$, and $\sigma_{32}=0.5$; oscillation appears under environmental driving forces which 
TABLE 1: Values of $\left(S_{1}^{*}, R_{1}^{*}\right)$, when fixing $\delta_{2}=0.012$ and changing $\delta_{1}$.

\begin{tabular}{lccc}
\hline$\delta_{1}=0.1$ & $\delta_{1}=0.025$ & $\delta_{1}=0.02$ & $\delta_{1}=0.01$ \\
$S_{1}^{*}=0.7627$ & $S_{1}^{*}=0.7290$ & $S_{1}^{*}=0.7263$ & $S_{1}^{*}=0.7205$ \\
$R_{1}^{*}=5.6130$ & $R_{1}^{*}=6.1694$ & $R_{1}^{*}=6.2105$ & $R_{1}^{*}=6.2945$ \\
\hline
\end{tabular}

TABle 2: Values of $\left(S_{2}^{*}, R_{2}^{*}\right)$, when fixing $\delta_{1}=0.01$ and changing $\delta_{2}$.

\begin{tabular}{lccc}
\hline$\delta_{2}=0.35$ & $\delta_{2}=0.25$ & $\delta_{2}=0.15$ & $\delta_{2}=0.012$ \\
$S_{2}^{*}=0.4678$ & $S_{2}^{*}=0.4502$ & $S_{2}^{*}=0.4254$ & $S_{2}^{*}=0.3663$ \\
$R_{2}^{*}=1.2710$ & $R_{2}^{*}=1.4692$ & $R_{2}^{*}=1.7408$ & $R_{2}^{*}=2.3383$ \\
\hline
\end{tabular}

actually affect the deterministic curves shown in Figure 5. But Figure 6 still maintains the same total trend as Figure 5 and reaches the same equilibrium point as deterministic version. Simulation result agrees with the real life situation.

Figure 7 and Tables 1 and 2 show that when the rate of immunity loss $\delta_{k}$ gradually reduces, $S_{k}^{*}$ decreases, but $R_{k}^{*}$ increases; the lower the rate of immunity loss $\delta_{k}$ is, the lower the steady state value of the susceptible is, the higher the steady state value of the recovered is. Thus, it will be of great importance for health management to control the rate of immunity loss to keep it in a lower level; for example, when antibody concentrations of recovered individuals are at a low level or are zero, they can be required to undergo vaccination to achieve the protective antibody levels.

\section{Conclusion}

This paper presented a mathematical study describing the dynamical behavior of an SIRS epidemic model with stochastic perturbations. Our purpose was based on analyzing this behavior using a stochastic model. When the reproduction number $\mathscr{R}_{0}$ is greater than one, we obtained sufficient conditions for stochastic stability of the endemic equilibrium $P^{*}$ by using a suitable Lyapunov function and other techniques of stochastic analysis. The investigation of this stochastic model revealed that the stochastic stability of $P^{*}$ depends on the magnitude of the intensity of noise as well as the parameters involved within the model system. Finally, numerical simulations are given to validate the theoretical results. The proposed model, a more accurate epidemic model, can help us to understand the dynamic behavior of virus. Moreover, the theoretical results may provide some useful guidance for making effective countermeasures on virus propagation.

\section{Conflict of Interests}

The author declares that there is no conflict of interests regarding the publication of this paper.

\section{Acknowledgment}

The author was partially supported by Project of Science and Technology of Heilongjiang Province of China no. 12531187.

\section{References}

[1] C. Ji, D. Jiang, and N. Shi, "Multigroup SIR epidemic model with stochastic perturbation," Physica A, vol. 390 , no. 10, pp. 17471762, 2011.

[2] A. Lajmanovich and J. A. Yorke, "A deterministic model for gonorrhea in a nonhomogeneous population," Mathematical Biosciences, vol. 28, no. 3-4, pp. 221-236, 1976.

[3] E. Beretta and V. Capasso, "Global stability results for a multigroup SIR epidemic model," in Mathematical Ecology, T. G. Hallam, L. J. Gross, and S. A. Levin, Eds., pp. 317-342, World Scientific, Teaneck, NJ, USA, 1988.

[4] H. W. Hethcote, "An immunization model for a heterogeneous population," Theoretical Population Biology, vol. 14, no. 3, pp. 338-349, 1978.

[5] H. W. Hethcote and H. R. Thieme, "Stability of the endemic equilibrium in epidemic models with subpopulations," Mathematical Biosciences, vol. 75, no. 2, pp. 205-227, 1985.

[6] H. R. Thieme, "Local stability in epidemic models for heterogeneous populations," in Mathematics in Biology and Medicine, V. Capasso, E. Grosso, and S. L. Paveri-Fontana, Eds., vol. 57 of Lecture Notes in Biomathematics, pp. 185-189, Springer, Berlin, Germany, 1985.

[7] H. R. Thieme, Mathematics in Population Biology, Princeton University Press, Princeton, NJ, USA, 2003.

[8] H. Guo, M. Y. Li, and Z. Shuai, "A graph-theoretic approach to the method of global Lyapunov functions," Proceedings of the American Mathematical Society, vol. 136, no. 8, pp. 2793-2802, 2008.

[9] M. Y. Li, Z. Shuai, and C. Wang, "Global stability of multigroup epidemic models with distributed delays," Journal of Mathematical Analysis and Applications, vol. 361, no. 1, pp. 3847, 2010.

[10] H. Guo, M. Y. Li, and Z. Shuai, "Global stability of the endemic equilibrium of multigroup SIR epidemic models," Canadian Applied Mathematics Quarterly, vol. 14, no. 3, pp. 259-284, 2006.

[11] Y. Muroya, Y. Enatsu, and T. Kuniya, "Global stability for a multi-group SIRS epidemic model with varying population sizes," Nonlinear Analysis: Real World Applications, vol. 14, no. 3, pp. 1693-1704, 2013.

[12] Y. Nakata, Y. Enatsu, and Y. Muroya, "On the global stability of an SIRS epidemic model with distributed delays," Discrete and Continuous Dynamical Systems A, vol. 2011, pp. 1119-1128, 2011.

[13] Y. Enatsu, Y. Nakata, and Y. Muroya, "Lyapunov functional techniques for the global stability analysis of a delayed SIRS epidemic model," Nonlinear Analysis: Real World Applications, vol. 13, no. 5, pp. 2120-2133, 2012.

[14] C. C. McCluskey, "Complete global stability for an SIR epidemic model with delay-distributed or discrete," Nonlinear Analysis: Real World Applications, vol. 11, no. 1, pp. 55-59, 2010.

[15] N. Dalal, D. Greenhalgh, and X. Mao, "A stochastic model of AIDS and condom use," Journal of Mathematical Analysis and Applications, vol. 325, no. 1, pp. 36-53, 2007.

[16] C. Ji, D. Jiang, and N. Shi, "Analysis of a predator-prey model with modified Leslie-Gower and Holling-type II schemes with stochastic perturbation," Journal of Mathematical Analysis and Applications, vol. 359, no. 2, pp. 482-498, 2009.

[17] C. Ji, D. Jiang, and X. Li, "Qualitative analysis of a stochastic ratio-dependent predator-prey system," Journal of Computational and Applied Mathematics, vol. 235, no. 5, pp. 1326-1341, 2011. 
[18] E. Tornatore, S. M. Buccellato, and P. Vetro, "Stability of a stochastic SIR system," Physica A, vol. 354, no. 1-4, pp. 111-126, 2005.

[19] E. Beretta, V. Kolmanovskii, and L. Shaikhet, "Stability of epidemic model with time delays influenced by stochastic perturbations," Mathematics and Computers in Simulation, vol. 45, no. 3-4, pp. 269-277, 1998.

[20] L. Shaikhet, "Stability of predator-prey model with aftereffect by stochastic perturbation," Stability and Control, vol. 1, no. 1, pp. 3-13, 1998.

[21] M. Carletti, "On the stability properties of a stochastic model for phage-bacteria interaction in open marine environment," Mathematical Biosciences, vol. 175, no. 2, pp. 117-131, 2002.

[22] M. Bandyopadhyay and J. Chattopadhyay, "Ratio-dependent predator-prey model: effect of environmental fluctuation and stability," Nonlinearity, vol. 18, no. 2, pp. 913-936, 2005.

[23] R. R. Sarkar and S. Banerjee, "Cancer self remission and tumor stability-a stochastic approach," Mathematical Biosciences, vol. 196, no. 1, pp. 65-81, 2005.

[24] M. Bandyopadhyay, T. Saha, and R. Pal, "Deterministic and stochastic analysis of a delayed allelopathic phytoplankton model within fluctuating environment," Nonlinear Analysis: Hybrid Systems, vol. 2, no. 3, pp. 958-970, 2008.

[25] L. Shaikhet, "Stability of a positive point of equilibrium of one nonlinear system with aftereffect and stochastic perturbations," Dynamic Systems and Applications, vol. 17, no. 1, pp. 235-253, 2008.

[26] N. Bradul and L. Shaikhet, "Stability of the positive point of equilibrium of Nicholson's blowflies equation with stochastic perturbations: numerical analysis," Discrete Dynamics in Nature and Society, vol. 2007, Article ID 92959, 25 pages, 2007.

[27] B. Mukhopadhyay and R. Bhattacharyya, "A nonlinear mathematical model of virus-tumor-immune system interaction: deterministic and stochastic analysis," Stochastic Analysis and Applications, vol. 27, no. 2, pp. 409-429, 2009.

[28] C. Yuan, D. Jiang, D. O'Regan, and R. P. Agarwal, “Stochastically asymptotically stability of the multi-group SEIR and SIR models with random perturbation," Communications in Nonlinear Science and Numerical Simulation, vol. 17, no. 6, pp. 2501-2516, 2012.

[29] J. Yu, D. Jiang, and N. Shi, "Global stability of two-group SIR model with random perturbation," Journal of Mathematical Analysis and Applications, vol. 360, no. 1, pp. 235-244, 2009.

[30] L. Imhof and S. Walcher, "Exclusion and persistence in deterministic and stochastic chemostat models," Journal of Differential Equations, vol. 217, no. 1, pp. 26-53, 2005.

[31] X. Fan and Z. Wang, "Stability analysis of an SEIR epidemic model with stochastic perturbation and numerical simulation," International Journal of Nonlinear Sciences and Numerical Simulation, vol. 14, no. 2, pp. 113-121, 2013.

[32] X. Fan, Z. Wang, and X. Xu, "Global stability of two-group epidemic models with distributed delays and random perturbation," Abstract and Applied Analysis, vol. 2012, Article ID 132095, 12 pages, 2012.

[33] Z. Wang, X. Fan, and Q. Han, "Global stability of deterministic and stochastic multigroup SEIQR models in computer network," Applied Mathematical Modelling, vol. 37, no. 20-21, pp. 8673-8686, 2013.

[34] X. Mao, Stochastic Differential Equations and Applications, Horwood Publishing, Chichester, UK, 2nd edition, 2008. 


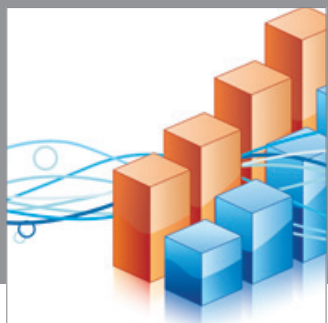

Advances in

Operations Research

mansans

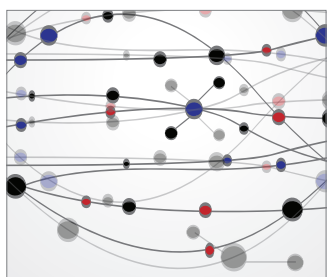

The Scientific World Journal
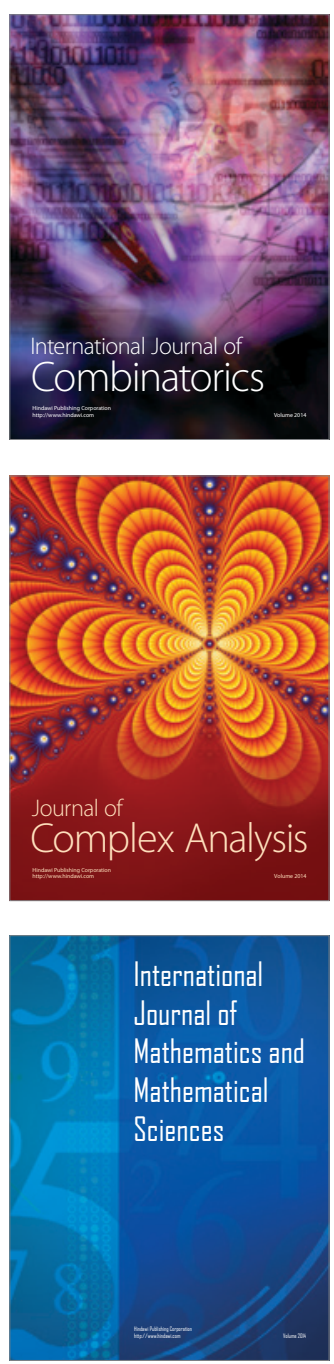
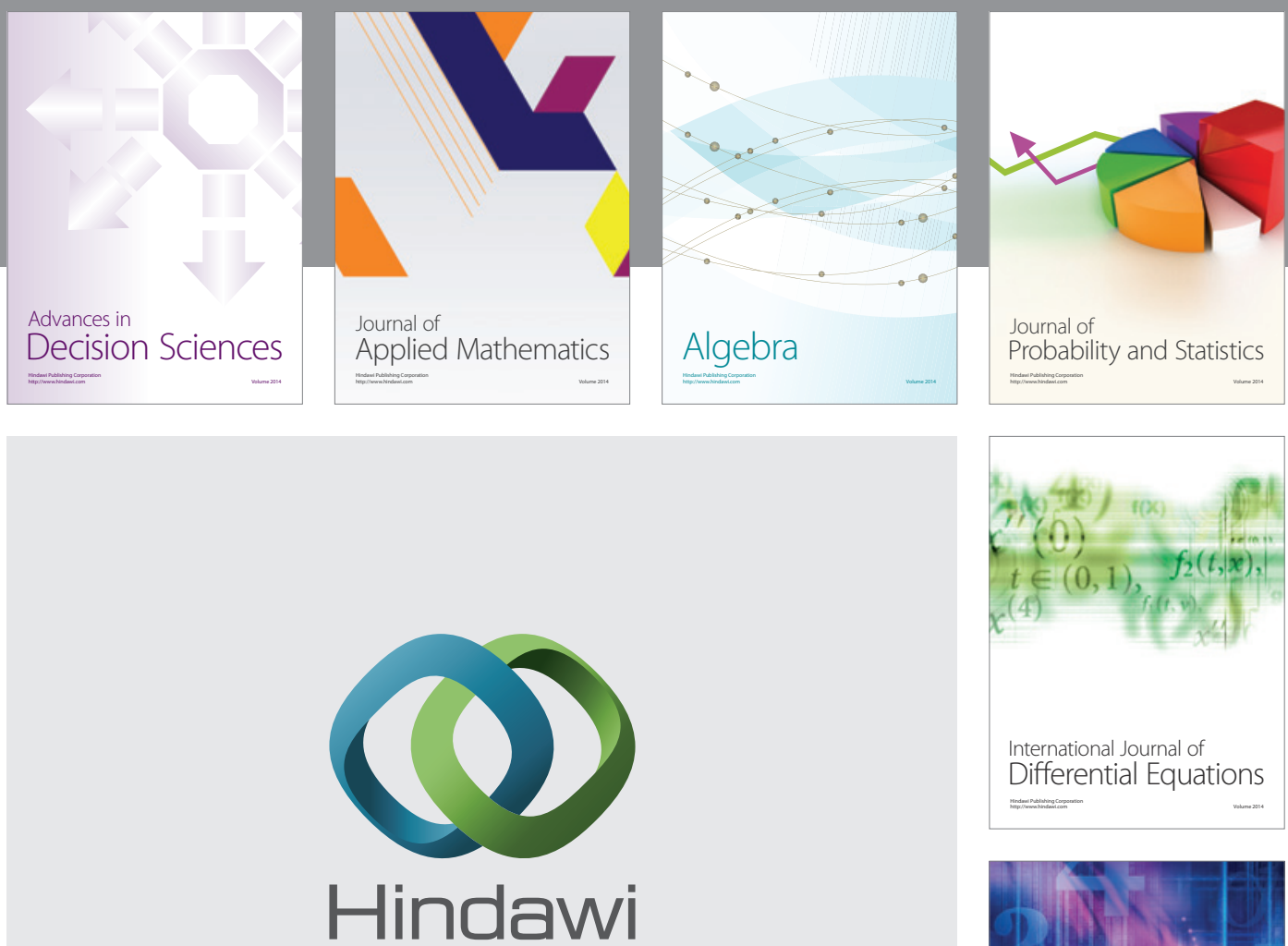

Submit your manuscripts at http://www.hindawi.com
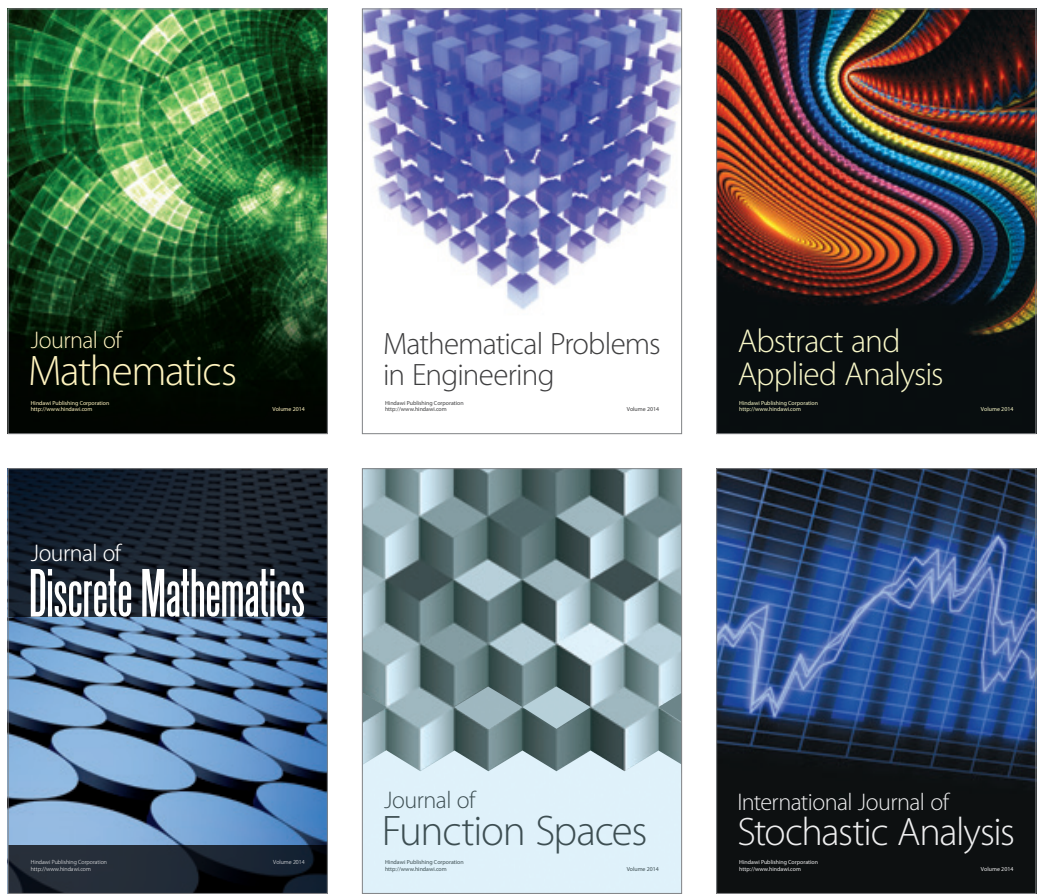

Journal of

Function Spaces

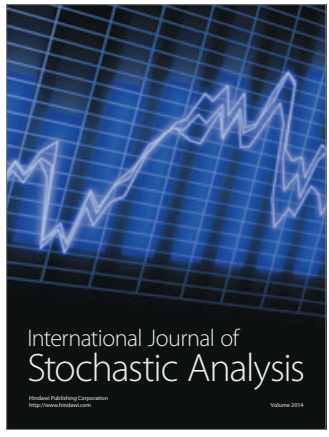

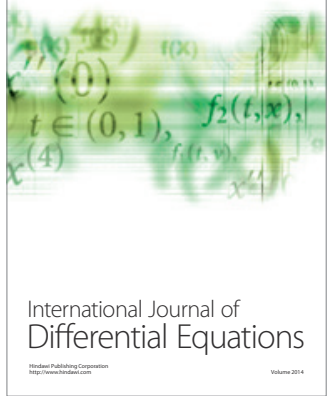
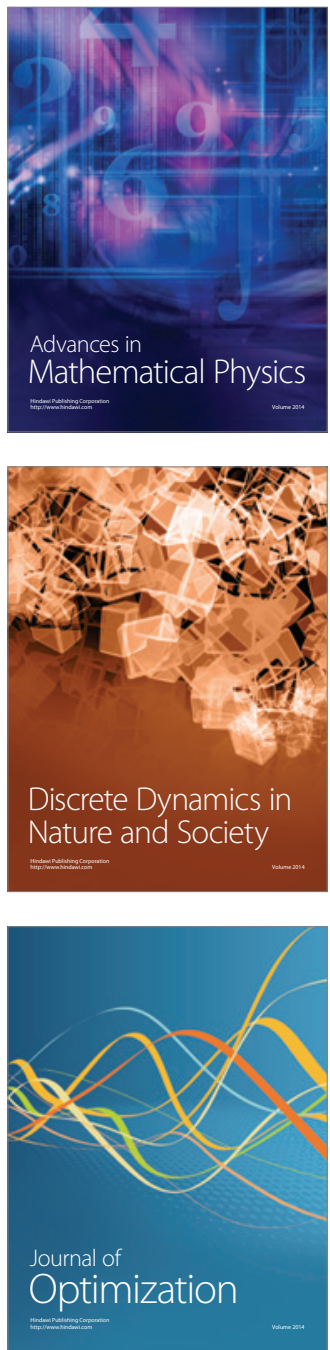